\title{
Constructing spatial discretizations for sparse multivariate trigonometric polynomials that allow for a fast discrete Fourier transform
}

\author{
Lutz Kämmerer* \\ July 16, 2018
}

\begin{abstract}
The paper discusses the construction of high dimensional spatial discretizations for arbitrary multivariate trigonometric polynomials, where the frequency support of the trigonometric polynomial is known. We suggest a construction based on the union of several rank-1 lattices as sampling scheme. We call such schemes multiple rank-1 lattices. This approach automatically makes available a fast discrete Fourier transform (FFT) on the data.

The key objective of the construction of spatial discretizations is the unique reconstruction of the trigonometric polynomial using the sampling values at the sampling nodes. We develop different construction methods for multiple rank-1 lattices that allow for this unique reconstruction. The symbol $M$ denotes the total number of sampling nodes within the multiple rank-1 lattice. In addition, we assume that the multivariate trigonometric polynomial is a linear combination of $T$ trigonometric monomials. The ratio of the number $M$ of sampling points that are sufficient for the unique reconstruction to the number $T$ of distinct monomials is called oversampling factor in this context. The presented construction methods for multiple rank-1 lattices allow for estimates of this number $M$. Roughly speaking, the oversampling factor $M / T$ is independent of the spatial dimension and, with high probability, only logarithmic in $T$, which is much better than the oversampling factor that is expected for a sampling method that uses one single rank-1 lattice.

The newly developed approaches for the construction of spatial discretizations are probabilistic methods. The arithmetic complexity of these algorithms depend only linearly on the spatial dimension and, with high probability, only linearly on $T$ up to some logarithmic factors.

Furthermore, we analyze the computational complexities of the resulting FFT algorithms, that exploits the structure of the suggested multiple rank-1 lattice spatial discretizations, in detail and obtain upper bounds in $\mathcal{O}(M \log M)$, where the constants depend only linearly on the spatial dimension. With high probability, we construct spatial discretizations where $M / T \leq C \log T$ holds, which implies that the complexity of the corresponding FFT converts to $\mathcal{O}\left(T \log ^{2} T\right)$.
\end{abstract}

Keywords and phrases : sparse multivariate trigonometric polynomials, lattice rule, multiple rank-1 lattice, fast Fourier transform

2010 AMS Mathematics Subject Classification : 60B15, 65T50,68Q25,68W40, 94A20

\footnotetext{
${ }^{*}$ Chemnitz University of Technology, Faculty of Mathematics, 09107 Chemnitz, Germany

kaemmerer@mathematik.tu-chemnitz.de
} 


\section{Introduction}

Since almost 60 years rank-1 lattice rules as Quasi-Monte Carlo type cubature rules were investigated in the field of numerical integration. An overview on the early work on lattice rules can be found in [17]. A lot of meaningful theoretical facts in the field of numerical integration could be proved using rank-1 lattices as sampling schemes. The crucial breakthrough for practical applications was the development of component-by-component constructions for rank-1 lattices. Under specific assumptions, this construction method is very practicable and the corresponding cubature rules guarantee optimal worst case error rates for the numerical integration of specific multivariate functions, cf., e.g., [3]. Already in the late 1950s, N. M. Korobov developed the ideas and a substantial theory on that topic in, e.g., [14, 15]. Unfortunately, N. M. Korobov published in Russian, which is at least one reason for the lack of awareness of his results. In 2002, the component-by-component idea was re-invented in [19]. This paper can be regarded as the initiation of the subsequent activities of a lot of researchers on component-by-component constructions.

The book of N. M. Korobov, cf. [15, Chapter IV], already contains estimates for the error of approximation methods based on sampling along single rank-1 lattices. The main focus was on functions of dominating mixed smoothness, which is still a highly topical research field. In the 1980's, V. N. Temlyakov [20] improves the results of N. M. Korobov using number theoretic argumentations, which lead to existence results but does not allow for the construction of suitable rank-1 lattices. Later, in $[16,12,13]$ similar considerations led to upper bounds on the worst case error in terms of the number of used sampling values, that were similar to those in [20] and still unsatisfactory in relation to, e.g., sparse grid approximation errors [4]. However, the results in $[16,12]$ provides practicable methods for the construction of suitable rank-1 lattices. A more recent paper, cf. [2], discusses the aforementioned non-optimal approximation errors in detail and presents a lower bound on the worst case approximation error for rank-1 lattice sampling that is essentially the same as the already known upper bound. Accordingly, the optimal worst case errors for approximation cannot even nearly be reached using rank-1 lattice sampling for specific approximation problems, e.g., hyperbolic cross approximations.

Nevertheless rank-1 lattices as sampling schemes provide stability, available efficient algorithms for computing the discrete Fourier transform [9], and good approximation properties, cf., e.g., [2], in particular for high dimensional approximation problems. For specific frequency sets $I \subset \mathbb{Z}^{d}$, the crucial disadvantage of rank-1 lattices is the large number $M$ of sampling values one necessarily needs in order to uniquely reconstruct a trigonometric polynomial with frequencies supported on the frequency set $I$. Depending on the structure of the frequency set $I$, the number $M$ is bounded from below by a term $M \geq C|I|^{2}$, cf. [9].

In order to overcome the limitations of the single rank-1 lattice approach, the author presented in [10] the idea to use multiple rank-1 lattices as spatial discretizations for multivariate trigonometric polynomials. A first simple construction method for multiple rank-1 lattices is published therein, where the construction is mainly based on the random determination of generating vectors of single rank-1 lattices. Various numerical experiments illustrate the advantages of these spatial discretizations, such as

- the existence of a fast discrete Fourier transform (FFT),

- low oversampling factors of sampling sets that allow for a unique reconstruction of multivariate trigonometric polynomials from their sampling values, 
- small condition numbers of the corresponding Fourier matrices,

- excellent approximation properties in specific problems.

Supposing that the above advantages actually hold in general — or at least with high probability - we will overcome limitations of sparse grid sampling as well as limitations of single rank-1 lattice sampling, which are unbounded condition numbers, cf. [11], and necessarily huge oversampling factors, cf. [9], respectively.

While the fast discrete Fourier transform algorithms are already presented in [10], all other above listed advantages are not proven yet.

In this paper, we incorporate ideas from [1] into the algorithm that is developed in $[10$, Alg. 5] such that the construction of multiple rank-1 lattices will allow for estimates of the sizes and the number of single rank-1 lattices that are joined to a spatial discretization.

We reflect some basics on multivariate trigonometric polynomials and (multiple) rank-1 lattices from $[9,10]$. At first, we define the torus $\mathbb{T} \simeq[0,1)$ and the multivariate trigonometric polynomial

$$
p: \mathbb{T}^{d} \rightarrow \mathbb{C}, \quad p(\boldsymbol{x}):=\sum_{\boldsymbol{k} \in I} \hat{p}_{\boldsymbol{k}} \mathrm{e}^{2 \pi \mathrm{i} \boldsymbol{k} \cdot \boldsymbol{x}},
$$

where $I \subset \mathbb{Z}^{d}$ is called frequency set, its cardinality $|I|<\infty$ is finite, and $\hat{p}_{\boldsymbol{k}}$ is named Fourier coefficient to the frequency $\boldsymbol{k}$ of the multivariate trigonometric polynomial $p$. For an arbitrary set of sampling nodes $\mathcal{X} \subset \mathbb{T}^{d},|\mathcal{X}|<\infty$, the Fourier matrix

$$
\boldsymbol{A}(\mathcal{X}, I):=\left(\mathrm{e}^{2 \pi \mathrm{i} \boldsymbol{k} \cdot \boldsymbol{x}}\right)_{\boldsymbol{x} \in \mathcal{X}, \boldsymbol{k} \in I}
$$

allows for the computation of the evaluation of $p$ at all nodes of $\mathcal{X}$ using the matrix vector product

$$
\boldsymbol{p}=\boldsymbol{A}(\mathcal{X}, I) \hat{\boldsymbol{p}},
$$

where $\boldsymbol{p}=(p(\boldsymbol{x}))_{\boldsymbol{x} \in \mathcal{X}}$ and $\hat{\boldsymbol{p}}=\left(\hat{p}_{\boldsymbol{k}}\right)_{\boldsymbol{k} \in I}$ are vectors that contain the sampling values of $p$ at all sampling nodes from $\mathcal{X}$ and the Fourier coefficients of $p$, respectively. At this point, we need to assume that the elements of $I$ and $\mathcal{X}$ are in a fixed order to interrelate the matrix with the vectors. Knowing the Fourier coefficients $\hat{p}_{\boldsymbol{k}}$, the matrix vector product will compute the sampling values of $p$ at all nodes of $\mathcal{X}$. On the other hand, we can also uniquely reconstruct the Fourier coefficients from known sampling values, iff the matrix $\boldsymbol{A}(\mathcal{X}, I)$ is of full column rank. In that case, the matrix $\boldsymbol{A}(\mathcal{X}, I)$ and, synonymously, the sampling scheme $\mathcal{X}$ allow for the unique reconstruction of all trigonometric polynomials $p \in \Pi_{I}:=\operatorname{span}\left\{\mathrm{e}^{2 \pi \mathrm{i} k \cdot \circ}: \boldsymbol{k} \in I\right\}$. Usually, one uses a normal equation $\hat{\boldsymbol{p}}=\left(\boldsymbol{A}(\mathcal{X}, I)^{*} \boldsymbol{A}(\mathcal{X}, I)\right)^{-1} \boldsymbol{A}(\mathcal{X}, I)^{*} \boldsymbol{p}$ in order to compute all Fourier coefficients $\hat{p}_{\boldsymbol{k}}, \boldsymbol{k} \in I$, of the trigonometric polynomial $p$, where $\boldsymbol{A}^{*}$ is the adjoint matrix of $\boldsymbol{A}$.

The central building block of our construction of spatial discretizations are so-called rank-1 lattices

$$
\Lambda(z, M)=\left\{\frac{j}{M} z \bmod \mathbf{1}: j=0, \ldots, M-1\right\} \subset \mathbb{T}^{d}
$$


where $\boldsymbol{z} \in \mathbb{Z}^{d}$ and $M \in \mathbb{N}$ are called generating vector and lattice size of $\Lambda(\boldsymbol{z}, M)$, respectively. The modulo 1 operation denotes a component-wise operation $x \bmod 1=x-\lfloor x\rfloor \in \mathbb{T}$ and implies that $\Lambda(\boldsymbol{z}, M) \subset[0,1)^{d}$ holds. A multiple rank-1 lattice

$$
\Lambda\left(\boldsymbol{z}_{1}, M_{1}, \ldots, \boldsymbol{z}_{s}, M_{s}\right):=\bigcup_{\ell=1}^{s} \Lambda\left(\boldsymbol{z}_{\ell}, M_{\ell}\right)
$$

is a union of a set of rank-1 lattices. Obviously, we obtain

$$
\left|\Lambda\left(\boldsymbol{z}_{1}, M_{1}, \ldots, \boldsymbol{z}_{s}, M_{s}\right)\right| \leq 1-s+\sum_{\ell=1}^{s} M_{\ell}
$$

where at least for the case that all $M_{\ell}$ are pairwise coprime and $\boldsymbol{z}_{\ell} \not \equiv \mathbf{0}\left(\bmod M_{\ell}\right), \ell=1, \ldots, s$, the equality holds, cf. [10, Cor. 2.3]. Since we use multiple rank-1 lattices as spatial discretization of multivariate trigonometric polynomials, we consider the corresponding Fourier matrix with $\mathcal{X}=\Lambda\left(\boldsymbol{z}_{1}, M_{1}, \ldots, \boldsymbol{z}_{s}, M_{s}\right)$

$$
\boldsymbol{A}:=\boldsymbol{A}\left(\Lambda\left(\boldsymbol{z}_{1}, M_{1}, \ldots, \boldsymbol{z}_{s}, M_{s}\right), I\right):=\left(\begin{array}{c}
\left(\mathrm{e}^{2 \pi \mathrm{i} \frac{j}{M_{1}} \boldsymbol{k} \cdot \boldsymbol{z}_{1}}\right)_{j=0, \ldots, M_{1}-1, \boldsymbol{k} \in I} \\
\left(\mathrm{e}^{2 \pi \mathrm{i} \frac{j}{M_{2}} \boldsymbol{k} \cdot \boldsymbol{z}_{2}}\right)_{j=1, \ldots, M_{2}-1, \boldsymbol{k} \in I} \\
\vdots \\
\left(\mathrm{e}^{2 \pi \mathrm{i} \frac{j}{M_{s}} \boldsymbol{k} \cdot \boldsymbol{z}_{s}}\right)_{j=1, \ldots, M_{s}-1, \boldsymbol{k} \in I}
\end{array}\right) .
$$

We stress on the fact, that this construction does not comply with the general construction in (1.1) since we allow for identical rows within $\boldsymbol{A}\left(\Lambda\left(\boldsymbol{z}_{1}, M_{1}, \ldots, \boldsymbol{z}_{s}, M_{s}\right), I\right)$ in the case that the equality in (1.2) does not hold. The crucial advantage of this construction of the Fourier matrix and the corresponding vector $\boldsymbol{p}$ as well is the simple application of $s$ rank-1 lattice FFTs, where each of them is the fast computation of a matrix vector product $\boldsymbol{A}\left(\Lambda\left(\boldsymbol{z}_{\ell}, M_{\ell}\right), I\right) \hat{\boldsymbol{p}}$, $\ell \in\{1, \ldots, s\}$, instead of using the matrix $\boldsymbol{A}\left(\Lambda\left(\boldsymbol{z}_{1}, M_{1}, \ldots, \boldsymbol{z}_{s}, M_{s}\right), I\right)$ for computing the sampling values of $p$ at all nodes within $\Lambda\left(\boldsymbol{z}_{1}, M_{1}, \ldots, \boldsymbol{z}_{s}, M_{s}\right)$. The corresponding complexity is in $\mathcal{O}(M \log M+s d|I|)$, where $M=\sum_{\ell=1}^{s} M_{\ell}$, cf. [10, Alg. 3].

Even the solution of the normal equation $\hat{\boldsymbol{p}}=\boldsymbol{A}^{\dagger} \boldsymbol{p}$, where $\boldsymbol{A}^{\dagger}:=\left(\boldsymbol{A}^{*} \boldsymbol{A}\right)^{-1} \boldsymbol{A}^{*}$ is the pseudo-inverse of $\boldsymbol{A}$, for given sampling values $\boldsymbol{p}$ can be efficiently computed by means of a conjugate gradient method that uses rank-1 lattice FFTs and its adjoint FFTs, cf. [10, Sec. 4]. Additional requirements on the multiple rank-1 lattice allow for a direct reconstruction method, cf. [10, Alg. 6], that has a total complexity in $\mathcal{O}(M \log M+s(d+\log |I|)|I|)$. At this point, we would like to emphasize that all constructions in the present paper, if successful, allow for the application of this direct fast reconstruction algorithm. We call a multiple rank-1 lattice that allows for a unique reconstruction of all trigonometric polynomials $p \in \Pi_{I}$, i.e., the matrix $\boldsymbol{A}\left(\Lambda\left(\boldsymbol{z}_{1}, M_{1}, \ldots, \boldsymbol{z}_{s}, M_{s}\right), I\right)$ has full column rank, reconstructing multiple rank-1 lattice for the frequency set $I$ or, synonymously, for all polynomials $p \in \Pi_{I}$.

Obviously, the aforementioned complexities are all bounded by terms that are at most linear in $M$ up to a logarithmic factor plus linear in $|I|$ up to a logarithmic factor, which is clearly much less than the complexity $\mathcal{O}(M|I|)$ of a matrix vector product using the Fourier matrix $\boldsymbol{A}\left(\Lambda\left(\boldsymbol{z}_{1}, M_{1}, \ldots, \boldsymbol{z}_{s}, M_{s}\right), I\right)$. Nevertheless, the reconstruction problem will only provide unique solutions if the Fourier matrix $\boldsymbol{A}$ is of full column rank, i.e., a unique 


\begin{tabular}{|c|c|c|c|}
\hline \multirow[b]{2}{*}{$I$} & \multicolumn{3}{|c|}{ computational complexities } \\
\hline & $\mathcal{X}$ & $\boldsymbol{A}(\mathcal{X}, I) \boldsymbol{p} \quad \boldsymbol{A}^{\dagger}(\mathcal{X}, I) \hat{\boldsymbol{p}}$ & references \\
\hline $\begin{array}{l}\text { full grid } \\
|I|=N^{d}\end{array}$ & $\begin{array}{l}\text { full grid } \\
|\mathcal{X}|=N^{d}\end{array}$ & $\mathcal{O}\left(N^{d} \log N\right)$ & {$[22]$} \\
\hline $\begin{array}{l}\text { hyperbolic cross } \\
|I| \simeq C_{d} N \log ^{d-1} N\end{array}$ & $\begin{array}{c}\text { sparse grid } \\
|\mathcal{X}| \simeq C_{d} N \log ^{d-1} N\end{array}$ & $\mathcal{O}\left(N \log ^{d} N\right)$ & {$[6]$} \\
\hline $\begin{array}{l}\text { hyperbolic cross } \\
|I| \simeq C_{d} N \log ^{d-1} N\end{array}$ & $\begin{array}{c}\text { rank-1 lattice } \\
|\mathcal{X}| \lesssim c_{d} N^{2} \log ^{d-2} N\end{array}$ & $\mathcal{O}\left(N^{2} \log ^{d-1} N\right)$ & {$[8]$} \\
\hline $\begin{array}{l}\text { hyperbolic cross } \\
|I| \simeq C_{d} N \log ^{d-1} N\end{array}$ & $\begin{array}{l}\text { multiple rank-1 lattice } \\
\qquad|\mathcal{X}| \lesssim c_{d} N \log ^{d} N\end{array}$ & $\mathcal{O}\left(N \log ^{d+1} N\right)$ & [10] \& Thm. 3.4 \\
\hline $\begin{array}{c}\text { arbitrary } \\
|I|=T, N_{I} \lesssim T\end{array}$ & $\begin{array}{c}\text { rank-1 lattice } \\
|\mathcal{X}|<T^{2}\end{array}$ & $\mathcal{O}\left(T^{2} \log T\right)$ & {$[9]$} \\
\hline $\begin{array}{l}\text { arbitrary } \\
|I|=T\end{array}$ & $\begin{array}{c}\text { random } \\
|\mathcal{X}| \lesssim T \log T\end{array}$ & $\mathcal{O}\left(T^{2} \log T\right)$ & {$[5]$} \\
\hline $\begin{array}{c}\text { arbitrary } \\
|I|=T, N_{I} \lesssim T\end{array}$ & $\begin{array}{l}\text { multiple rank-1 lattice } \\
\qquad|\mathcal{X}| \lesssim T \log T\end{array}$ & $\mathcal{O}\left(T \log ^{2} T\right)$ & [10] \& Thm. 3.4 \\
\hline
\end{tabular}

Table 1.1: Different types of frequency sets and corresponding spatial discretization schemes in comparison. In addition, the computational complexities of known evaluation and reconstruction algorithms and references.

reconstruction may require a huge number of sampling values, cf. e.g. [9], where we proved that $M \sim|I|^{2}$ is necessary for a single rank-1 lattice as spatial discretization $\mathcal{X}$, which can be interpreted as the currently considered approach with $s=1$, in the worst case.

In this paper, we present algorithms that determine reconstructing multiple rank-1 lattices for given frequency sets $I$. The crucial difference to the already published algorithm, cf. [10, Alg. 5], is that the new algorithms allow for estimates on the total number of sampling nodes of the multiple rank-1 lattices that arise. Under the weak assumption that the frequency set $I \subset$ $\mathbb{Z}^{d},|I|>3$, is contained in a box of edge length $2(|I|-1)$, we prove that there exists at least one reconstructing multiple rank-1 lattice for $I$ with a number of sampling nodes that is bounded by $C|I| \ln |I|$, where $C<10$ is a fixed constant. Moreover, the presented algorithms are based on the theoretical considerations and their constructive proofs. The determined reconstructing multiple rank-1 lattices for frequency sets $I$ contain at most $C_{\delta}|I| \log |I|$ sampling nodes with probability at least $1-\delta$, where $C_{\delta} \lesssim \log \delta$.

The last-mentioned constants $C$ and $C_{\delta}$ do not depend on the dimension $d$. If we assume the cardinality $M$ of the reconstructing multiple rank-1 lattice for $I$ fulfills $M \leq C|I| \ln |I|$, we achieve computational complexities in $\mathcal{O}(|I|(d+\log |I|) \log |I|)$ of the fast algorithms computing the matrix vector products $\boldsymbol{A} \hat{\boldsymbol{p}}$ and $\left(\boldsymbol{A}^{*} \boldsymbol{A}\right)^{-1} \boldsymbol{A}^{*} \boldsymbol{p}$, cf. [10]. At this point, we stress on the dimension $d$, that is only a linear factor, and the cardinality of the frequency set $I$, that arises as a linear factor times a polynomial of degree two in $\log |I|$.

In Table 1.1 we present different types of frequency sets $I$ and corresponding spatial discretization schemes $\mathcal{X}$ and also the computational complexity of known fast algorithms for the computation of the evaluation $\boldsymbol{p}=\boldsymbol{A} \hat{\boldsymbol{p}}$ and the reconstruction $\hat{\boldsymbol{p}}=\boldsymbol{A}^{\dagger} \boldsymbol{p}$ of the corresponding multivariate trigonometric polynomials in order to classify the result of this paper. We stress the fact that we have left out terms that depend only on $d$ in the computational complexities 
since they are not analyzed in specific cases.

The full grid case is well known as FFT of multidimensional arrays and only provided as reference in the table. Considering hyperbolic crosses as frequency sets, there already exist two approaches for spatial discretizations that allow for a fast Fourier transform. First, sparse grids as spatial discretizations and the corresponding hyperbolic cross FFT provide a complexity that is almost linear in the number of frequencies up to a logarithmic factor. Unfortunately, the associated discrete Fourier transform is not stable, cf. [11]. Second, a single rank-1 lattice discretization for hyperbolic cross trigonometric polynomials requires at least $N^{2}$ sampling nodes and we are able to construct a single rank-1 lattice of size $c_{d} N^{2} \log ^{d-2} N$ that allows for a unique reconstruction of hyperbolic cross trigonometric polynomials, cf. [9]. The complexity of the corresponding fast Fourier transform is linear in the number of sampling nodes within the spatial discretization up to a logarithmic factor. The bottleneck of this approach is the necessarily huge number of sampling nodes of the spatial discretization.

In this paper, we show that the concept of multiple rank-1 lattices allows for spatial discretizations of hyperbolic cross trigonometric polynomials of much lower cardinality than single rank-1 lattices, i.e., we prove oversampling factors that are bounded by $C \log N$. For single rank-1 lattices we observed oversampling factors of approximately $C N / \log N$. Regarding only the oversampling of the last mentioned spatial discretizations for hyperbolic cross trigonometric polynomials, we should prefer sparse grids. However, a critical look at the numerical tests and, in particular, on the condition numbers of the Fourier matrices may lead to another assessment, cf. Section 5.3. Apart from this, the general concept of sparse grids requires a specific kind of structure of the frequency sets $I$ called "downward closed". If this structure is seriously violated the sparse grid approach is not successfully applicable.

Last, Table 1.1 shows results for trigonometric polynomials with arbitrary frequency sets $I$ of cardinality $T$, where the listed sampling methods based on structured sampling sets, i.e., the single rank-1 lattice approach as well as the multiple rank-1 lattice approach, require some restriction on the expansion $N_{I}$, cf. (2.3), of the frequency set $I$ under consideration. A spatial discretization using a single rank-1 lattice structure needs less than $T^{2}$ sampling nodes and more than $c T^{2}, c>0$, sampling nodes in the worst case. The corresponding fast Fourier transform is linear in the number of used sampling nodes up to a logarithmic factor. Here again, the number of necessary sampling nodes is the bottleneck of the single rank-1 lattice approach. On the contrary, random sampling requires only a few oversampling for stable Fourier matrices. Since the corresponding Fourier matrices suffer from a lack of structure, the discrete Fourier transform is realized using the matrix vector product and, if necessary, a conjugate gradient method. Hence, the computation is not efficient. The present paper shows that a multiple rank-1 lattice discretization involves oversampling that is similar in terms of $T$ to the oversampling that arises at random sampling. The fast Fourier transform algorithms in [10] exploit the structure of multiple rank-1 lattices and yield lower computational complexities compared to a random sampling approach.

The remainder of this paper is structured as follows. In Section 2, we collect some general basic facts about spatial discretizations of multivariate trigonometric polynomials. Subsequently, we present a simple probabilistic strategy that allows for the construction of spatial discretizations of multivariate trigonometric polynomials in Section 3. In particular, carefully chosen lattice sizes $M_{1}, \ldots, M_{s}$ and randomly chosen generating vectors $\boldsymbol{z}_{\ell} \in\left[0, M_{\ell}-1\right]^{d} \cap \mathbb{Z}^{d}$, $\ell=1, \ldots, s$, allow for an estimate of the success probability of this approach. In addition, we give upper bounds on the number $s$ of such rank-1 lattices that need to be joined in order to obtain - with a certain probability - specific properties of the resulting multiple rank-1 lattice 
that ensure the required full column rank of the Fourier matrix. We use these bounds on $s$ in order to estimate the number of sampling nodes within the constructed spatial discretizations. In Algorithms 1 and 2, we present an almost non-intrusive approach, i.e., only three basic facts, the dimension $d$, an upper bound $N$ on the expansion $N_{I}$, and an upper bound $T$ on the cardinality $|I|$ of the frequency set $I$, need to be known in order to construct a spatial discretization with high probability. The knowledge about the concrete locations of the elements in the frequency set $I$ allow for some slight modifications on the already developed algorithms and will decrease the number of used lattices and, with this, the number of sampling nodes within the spatial discretizations in practice, cf. Algorithms 3 and 4. In Section 4, we discuss some improvement ideas on the construction methods. We develop Algorithms 5 and 6 that are based on an iterative application of the ideas from Section 3 which allows for the reduction of the lattice sizes $M_{\ell}$ and leads to slightly larger theoretical bounds on the number $s$ of joined rank-1 lattices. Moreover, we present an approach, cf. Algorithm 7, that successively constructs spatial discretizations for parts of the frequency set $I$ under consideration which has the advantage that the used lattice sizes $M_{\ell}$ may be further reduced. In Section 5 , we present extensive numerical tests that confirm the theoretical results. Moreover, we observe stability of the spatial discretizations, i.e., the numerically determined condition numbers of the Fourier matrices $\boldsymbol{A}$, cf. (1.3), are outstandingly small.

\section{Basics}

We collect some basic observations about sampling schemes, first for multivariate trigonometric monomials and second for multivariate trigonometric polynomials with frequencies supported on shifted frequency sets.

Lemma 2.1. We consider a trigonometric polynomial consisting of at most one scaled monomial, i.e., $p(\boldsymbol{x})=\hat{p}_{\boldsymbol{k}} \mathrm{e}^{2 \pi \mathrm{i} \boldsymbol{k} \cdot \boldsymbol{x}}$. The corresponding frequency set $I \subset \mathbb{Z}^{d}$ contains one element $\boldsymbol{k} \in I$. Then, the single Fourier coefficient $\hat{p}_{\boldsymbol{k}}, \boldsymbol{k} \in I$, can be reconstructed using an arbitrary sampling set that consists of at least one sampling node $\boldsymbol{x}_{0} \in \mathbb{T}^{d}$.

Proof. Due to the fact that $\mathrm{e}^{2 \pi \mathrm{i} \boldsymbol{k} \cdot \boldsymbol{x}_{0}} \neq 0$, we achieve $\hat{p}_{\boldsymbol{k}}=\frac{p\left(\boldsymbol{x}_{0}\right)}{\mathrm{e}^{2 \pi \mathrm{i} k} \cdot \boldsymbol{x}_{0}}$.

Lemma 2.2. Let the numbers $a_{j}, b_{j} \in \mathbb{Z}$ with $a_{j} \leq b_{j}, j=1, \ldots, d$, be given. We consider the frequency set $I \subset\left\{a_{1}, \ldots, b_{1}\right\} \times \cdots \times\left\{a_{d}, \ldots, b_{d}\right\} \subset \mathbb{Z}^{d}$ and a sampling set $\mathcal{X}$ that allow for the unique reconstruction of all trigonometric polynomials supported on the frequency set $I_{0}:=\left\{\boldsymbol{k} \in \mathbb{Z}^{d}: \boldsymbol{k}=\boldsymbol{h}-\boldsymbol{a}, \boldsymbol{h} \in I\right\}, \boldsymbol{a}=\left(a_{1}, \ldots, a_{d}\right)^{\top}$. Then, sampling along the sampling set $\mathcal{X}$ also allows for the unique reconstruction of all trigonometric polynomials within $\Pi_{I}$.

Proof. We consider the matrix

$$
\begin{aligned}
\boldsymbol{A}\left(\mathcal{X}, I_{0}\right) & =\left(\mathrm{e}^{2 \pi \mathrm{i} \boldsymbol{k} \cdot \boldsymbol{x}_{j}}\right)_{\boldsymbol{k} \in I_{0}, \boldsymbol{x}_{j} \in \mathcal{X}}=\left(\mathrm{e}^{2 \pi \mathrm{i}(\boldsymbol{h}-\boldsymbol{a}) \cdot \boldsymbol{x}_{j}}\right)_{\boldsymbol{h}-\boldsymbol{a} \in I_{0}, \boldsymbol{x}_{j} \in \mathcal{X}} \\
& =\left(\mathrm{e}^{-2 \pi \mathrm{i} \boldsymbol{a} \cdot \boldsymbol{x}_{j}} \mathrm{e}^{2 \pi \mathrm{i} \boldsymbol{h} \cdot \boldsymbol{x}_{j}}\right)_{\boldsymbol{h} \in I, \boldsymbol{x}_{j} \in \mathcal{X}}=\boldsymbol{D}\left(\mathrm{e}^{2 \pi \mathrm{i} \boldsymbol{h} \cdot \boldsymbol{x}_{j}}\right)_{\boldsymbol{h} \in I, \boldsymbol{x}_{j} \in \mathcal{X}}=\boldsymbol{D} \boldsymbol{A}(\mathcal{X}, I),
\end{aligned}
$$

where $\boldsymbol{D}:=\operatorname{diag}\left(\mathrm{e}^{-2 \pi \mathrm{i} \boldsymbol{a} \cdot \boldsymbol{x}_{j}}\right)_{\boldsymbol{x}_{j} \in \mathcal{X}}$. The matrix $\boldsymbol{D}$ is of full rank. Consequently, the full column rank of $\boldsymbol{A}\left(\mathcal{X}, I_{0}\right)$ implies a full column rank of $\boldsymbol{A}(\mathcal{X}, I)$. 
Similar argumentations yield the general result, that shifting the frequency set does not affect the reconstruction property of a sampling set. Moreover, for the specific structure of rank-1 lattices $\Lambda(\boldsymbol{z}, M)$ we can show that even the modulo operation applied to each component of the vectors within $I$ does not affect the reconstruction property of the sampling set $\Lambda(\boldsymbol{z}, M)$ provided that the modulo operation on the frequencies in $I$ does not lead to collisions, i.e., the inequalities

$$
\boldsymbol{h} \bmod M \not \equiv \boldsymbol{k} \bmod M
$$

hold for all $\boldsymbol{h}, \boldsymbol{k} \in I, \boldsymbol{h} \neq \boldsymbol{k}$. In this context the modulo operation on vectors $\boldsymbol{k}$ is given by

$$
\boldsymbol{k} \bmod M:=\left(\begin{array}{c}
k_{1} \bmod M \\
\vdots \\
k_{d} \bmod M
\end{array}\right)
$$

where $k \bmod M=\min \left\{l \in \mathbb{N}_{0}: l \equiv k(\bmod M)\right\}$ is the unique smallest non-negative integer representative of the residue class of $k$ modulo $M$.

Lemma 2.3. We consider the frequency set $I \subset \mathbb{Z}^{d},|I|<\infty$, and we fix a prime number $M$. In addition, we define the frequency set

$$
I_{\bmod M}:=\left\{\boldsymbol{h}_{\boldsymbol{k}}:=\boldsymbol{k} \bmod M: \boldsymbol{k} \in I\right\}
$$

and choose a generating vector $\boldsymbol{z} \in[0, M-1]^{d} \cap \mathbb{Z}^{d}$ at random. If the equality $\left|I_{\bmod M}\right|=|I|$ holds, then the rank-1 lattice $\Lambda(\boldsymbol{z}, M)$ allows for the unique reconstruction of $\hat{p}_{\boldsymbol{k}}$ for $p \in \Pi_{I}$ iff the rank-1 lattice $\Lambda(\boldsymbol{z}, M)$ allows for the unique reconstruction of $\hat{\tilde{p}}_{\boldsymbol{h}_{\boldsymbol{k}}}$ for $\tilde{p} \in \Pi_{I_{\bmod M}}$.

Proof. Since we assume $|I|=\left|I_{\bmod M}\right|$, we find for each $\boldsymbol{k} \in I$ unique vectors $\boldsymbol{a}_{\boldsymbol{k}} \in \mathbb{Z}^{d}$ and $\boldsymbol{h}_{\boldsymbol{k}} \in[0, M-1]^{d} \cap \mathbb{Z}^{d}$ such that

$$
k:=M a_{k}+h_{k},
$$

where $\boldsymbol{h}_{\boldsymbol{k}_{1}} \neq \boldsymbol{h}_{\boldsymbol{k}_{2}}$ for all $\boldsymbol{k}_{1}, \boldsymbol{k}_{2} \in I, \boldsymbol{k}_{1} \neq \boldsymbol{k}_{2}$. The set $I_{\bmod M} \subset[0, M-1]^{d}$ is given by $I_{\bmod M}=\left\{\boldsymbol{h}_{\boldsymbol{k}}: \boldsymbol{k} \in I\right\}$. The corresponding Fourier matrices read as

$$
\begin{aligned}
\boldsymbol{A}\left(\Lambda(\boldsymbol{z}, M), I_{\bmod M}\right) & =\left(\mathrm{e}^{2 \pi \mathrm{i} \boldsymbol{h}_{\boldsymbol{k}} \cdot \boldsymbol{z} \frac{j}{M}}\right)_{\boldsymbol{h}_{\boldsymbol{k}} \in I_{\bmod M}, j=0, \ldots, M-1} \\
& =\left(\mathrm{e}^{2 \pi \mathrm{i}\left(\boldsymbol{h}_{\boldsymbol{k}} \cdot \boldsymbol{z} \frac{j}{M}+\frac{M j}{M} \boldsymbol{a}_{\boldsymbol{k}} \cdot \boldsymbol{z}\right)}\right)_{\boldsymbol{h}_{\boldsymbol{k}} \in I_{\bmod M}, j=0, \ldots, M-1} \\
& =\left(\mathrm{e}^{2 \pi \mathrm{i} \boldsymbol{k} \cdot \boldsymbol{z} \frac{j}{M}}\right)_{\boldsymbol{k} \in I, j=0, \ldots, M-1}=\boldsymbol{A}(\Lambda(\boldsymbol{z}, M), I),
\end{aligned}
$$

which yields the assertion.

Due to the last result, we collect all prime numbers $M$ such that $\left|I_{\bmod M}\right|=|I|$ holds. The set of these prime numbers is denoted by

$$
P^{I}:=\left\{M \in \mathbb{N}: M \text { prime with }\left|I_{\bmod M}\right|=|I|\right\} .
$$

In addition, we define the expansion of a frequency set $I$ by

$$
N_{I}:=\max _{j=1, \ldots, d}\left\{\max _{\boldsymbol{k} \in I} k_{j}-\min _{\boldsymbol{l} \in I} l_{j}\right\} .
$$

In fact, the number $N_{I}$ is the smallest number $N$ such that we can shift the set $I$ in $[0, N]^{d}$. Moreover, we observe the next Lemma. 
Lemma 2.4. The equality $\left|I_{\bmod M}\right|=|I|$ is satisfied for all $M \in \mathbb{N}, M>N_{I}$.

Proof. We assume $M>N_{I}$ with $\left|I_{\bmod } M\right|<|I|$, which implies that there is at least a pair $\boldsymbol{k}, \boldsymbol{l} \in I, \boldsymbol{k} \neq \boldsymbol{l}$, with $\boldsymbol{h}_{\boldsymbol{k}}=\boldsymbol{k} \bmod M=\boldsymbol{l} \bmod M=\boldsymbol{h}_{\boldsymbol{l}}$. According to $0 \neq \boldsymbol{k}-\boldsymbol{l}$ and $\boldsymbol{k}-\boldsymbol{l} \equiv \mathbf{0}$ $\bmod M$, there exists at least one $j_{0} \in\{1, \ldots, d\}$ with $\left|k_{j_{0}}-l_{j_{0}}\right|=\ell M \geq M>N_{I}, \ell \in \mathbb{N}$, which contradicts the definition of $N_{I}$.

Lemma 2.5. The set of prime numbers $P^{I}$ contains all prime numbers larger than $N_{I}$.

Proof. See Lemma 2.4.

\section{Generate reconstructing multiple rank-1 lattices}

We consider a frequency set $I \subset \mathbb{Z}^{d}$ of finite cardinality $T=|I|$ and fix one element $\boldsymbol{k} \in I$. The probability that another element $\boldsymbol{h} \in I \backslash\{\boldsymbol{k}\}$ aliases to this specific $\boldsymbol{k}$, while sampling along a random rank-1 lattice is estimated in the next lemma.

Lemma 3.1. We fix a frequency $\boldsymbol{k} \in I \subset \mathbb{Z}^{d},|I|=T<\infty$, and a prime number $M$ such that $\left|I_{\bmod M}\right|=|I|$, cf. (2.1). In addition, we choose a generating vector $\boldsymbol{z} \in[0, M-1]^{d}$ at random. Then, with probability of at most $\frac{T-1}{M}$ the frequency $\boldsymbol{k}$ aliases to at least one other frequency $\boldsymbol{h} \in I \backslash\{\boldsymbol{k}\}$.

Proof. First we take advantage of $\left|I_{\bmod M}\right|=|I|$ and determine the probability that one specific $\boldsymbol{h}_{\boldsymbol{k}^{\prime}} \in I_{\bmod M} \backslash\left\{\boldsymbol{h}_{\boldsymbol{k}}\right\}$ aliases to the fixed $\boldsymbol{h}_{\boldsymbol{k}}$, i.e.,

$$
\left(\boldsymbol{h}_{\boldsymbol{k}}-\boldsymbol{h}_{\boldsymbol{k}^{\prime}}\right) \cdot \boldsymbol{z} \equiv 0 \bmod M
$$

holds. Since $\boldsymbol{k} \neq \boldsymbol{k}^{\prime}$ and $\boldsymbol{h}_{\boldsymbol{k}} \neq \boldsymbol{h}_{\boldsymbol{k}^{\prime}}$ there exists a dimension index $r$ such that $h_{\boldsymbol{k}, r} \neq h_{\boldsymbol{k}^{\prime}, r}$. For any choice of the $d-1$ components $z_{1}, \ldots, z_{r-1}, z_{r+1}, \ldots, z_{d}$ of the generating vector $\boldsymbol{z}$, we determine exactly one $z_{r}$ with

$$
[0, M-1] \ni z_{r}=b \sum_{\substack{u=1 \\ u \neq r}}^{d}\left(h_{\boldsymbol{k}, u}-h_{\boldsymbol{k}^{\prime}, u}\right) z_{u} \bmod M,
$$

where $b:=\left\{t \in[1, M-1] \cap \mathbb{N}:\left(h_{\boldsymbol{k}^{\prime}, r}-h_{\boldsymbol{k}, r}\right) t \equiv 1(\bmod M)\right\}$ is the uniquely defined multiplicative inverse of $h_{\boldsymbol{k}^{\prime}, r}-h_{\boldsymbol{k}, r}$ modulo $M$. Accordingly, we observe (3.1). Consequently, a proportion of $1 / M$ choices of vectors $\boldsymbol{z}$ will cause an aliasing of the frequencies $\boldsymbol{h}_{\boldsymbol{k}}$ and $\boldsymbol{h}_{\boldsymbol{k}^{\prime}}$ or $\boldsymbol{k}$ and $\boldsymbol{k}^{\prime}$, cf. Lemma 2.3. By the union bound, the probability that a vector $\boldsymbol{z}$, that is uniformly chosen at random from $[0, M-1]^{d} \cap \mathbb{Z}^{d}$, yields an aliasing of the frequency $\boldsymbol{k}$ to at least one other frequency within $I$ is bounded from above by $\frac{T-1}{M}$.

Using a specific number of rank-1 lattices as sampling scheme provides for the unique reconstruction of the trigonometric polynomial $p \in \Pi_{I}$ with a certain probability, which can be beneficially estimated by the aid of Hoeffding's inequality [7].

Theorem 3.2. We consider the frequency set $I \subset \mathbb{Z}^{d}$ of cardinality $T$ and fix an element $\boldsymbol{k} \in I$. In addition, we fix $\delta \in(0,1)$ and we determine two numbers

$$
\begin{aligned}
& \lambda \geq c(T-1), \quad c>1, \\
& s=\left\lceil\left(\frac{c}{c-1}\right)^{2} \frac{\ln T-\ln \delta}{2}\right\rceil
\end{aligned}
$$


and the set of the $n \in \mathbb{N}$ smallest prime numbers in $P^{I}$, cf. (2.2), larger than $\lambda$

$$
P_{\lambda, n}^{I}:=\left\{p_{j} \in P^{I}: p_{j}=\left\{\begin{array}{ll}
\min \left\{p \in P^{I}: p>\lambda\right\} & : j=1 \\
\min \left\{p \in P^{I}: p>p_{j-1}\right\} & : j=2, \ldots, n .
\end{array}\right\}\right.
$$

We choose $s$ numbers $M_{\ell}, \ell=1, \ldots, s$, randomly from $P_{\lambda, n}^{I}$. For each of the numbers $M_{\ell}$, which are not necessarily distinct, we choose a generating vector $\boldsymbol{z}_{\ell} \in\left[0, M_{\ell}-1\right]^{d}$ at random. Then, the probability that the frequency $\boldsymbol{k} \in I$ aliases to any other frequency within I for each rank-1 lattice $\Lambda\left(\boldsymbol{z}_{\ell}, M_{\ell}\right)$ is bounded from above by $\frac{\delta}{T}$.

Proof. For the fixed frequency $\boldsymbol{k} \in I$, we define the random variables

$$
Y_{\ell}^{\boldsymbol{k}}:= \begin{cases}0 & : \boldsymbol{k} \text { does not alias to another frequency within } I \text { using } \Lambda\left(\boldsymbol{z}_{\ell}, M_{\ell}\right) \\ 1 & : \boldsymbol{k} \text { aliases to at least one other frequency within } I \text { using } \Lambda\left(\boldsymbol{z}_{\ell}, M_{\ell}\right)\end{cases}
$$

The random variables $Y_{\ell}^{k}, \ell=1, \ldots, s$, are independent and identically distributed with a specific mean $\mu \leq \frac{T-1}{\min _{p \in P_{\lambda, n}^{I}} p}<\frac{T-1}{\lambda} \leq \frac{1}{c}$, due to Lemma 3.1. Hoeffding's inequality allows for the estimate

$$
\begin{aligned}
\mathcal{P}\left\{\sum_{\ell=1}^{s} Y_{\ell}^{k}=s\right\} & =\mathcal{P}\left\{s^{-1} \sum_{\ell=1}^{s} Y_{\ell}^{\boldsymbol{k}}-\mu=1-\mu\right\} \leq \mathcal{P}\left\{s^{-1} \sum_{\ell=1}^{s} Y_{\ell}^{\boldsymbol{k}}-\mu \geq 1-\varepsilon-\mu\right\} \\
& \leq \mathrm{e}^{-2 s(1-\varepsilon-\mu)^{2}}=\mathrm{e}^{-2 s\left(\frac{c-1}{c}\right)^{2}} \leq \mathrm{e}^{\ln \delta-\ln T}=\frac{\delta}{T}
\end{aligned}
$$

for the specific choice $\varepsilon=\frac{1}{c}-\mu>0$.

If we choose the rank-1 lattice sizes $M_{1}, \ldots, M_{s}$ relatively prime, the resulting multiple rank-1 lattice $\Lambda\left(\boldsymbol{z}_{1}, M_{1}, \ldots, \boldsymbol{z}_{s}, M_{s}\right)$ is actually a structured subsampling of the rank-1 lattice $\Lambda\left(\boldsymbol{z}^{\prime}, M^{\prime}\right)$, where the generating vector and the lattice size are given by $\boldsymbol{z}^{\prime}=\sum_{r=1}^{s}\left(\prod_{\substack{l=1 \\ l \neq r}}^{s} M_{l}\right) \boldsymbol{z}_{r}$ and $M^{\prime}=\prod_{r=1}^{s} M_{r}$, respectively, cf. [10, Cor. 2.2]. Since specific properties of rank-1 lattices are extensively investigated, our studies on multiple rank-1 lattices as subsampling schemes of single rank-1 lattices may profit from this. On this account, we modify the results from Theorem 3.2 to these requirements.

Corollary 3.3. Doing the same as in Theorem 3.2 but without replacement of the $M_{\ell}$, i.e., the used $M_{\ell}$ are pairwise distinct which requires $n \geq s$, we get the analogous result.

Proof. Due to [7, Theorem 4] the bounds on probabilities for random samples without replacement coincide with those of similar random variables with replacement.

Another simple conclusion allows for the estimate of the probability that we can uniquely determine all Fourier coefficients $\hat{p}_{\boldsymbol{k}}, \boldsymbol{k} \in I$, from the sampling values at the nodes of the multiple rank-1 lattice constructed by the approach of Theorem 3.2. 


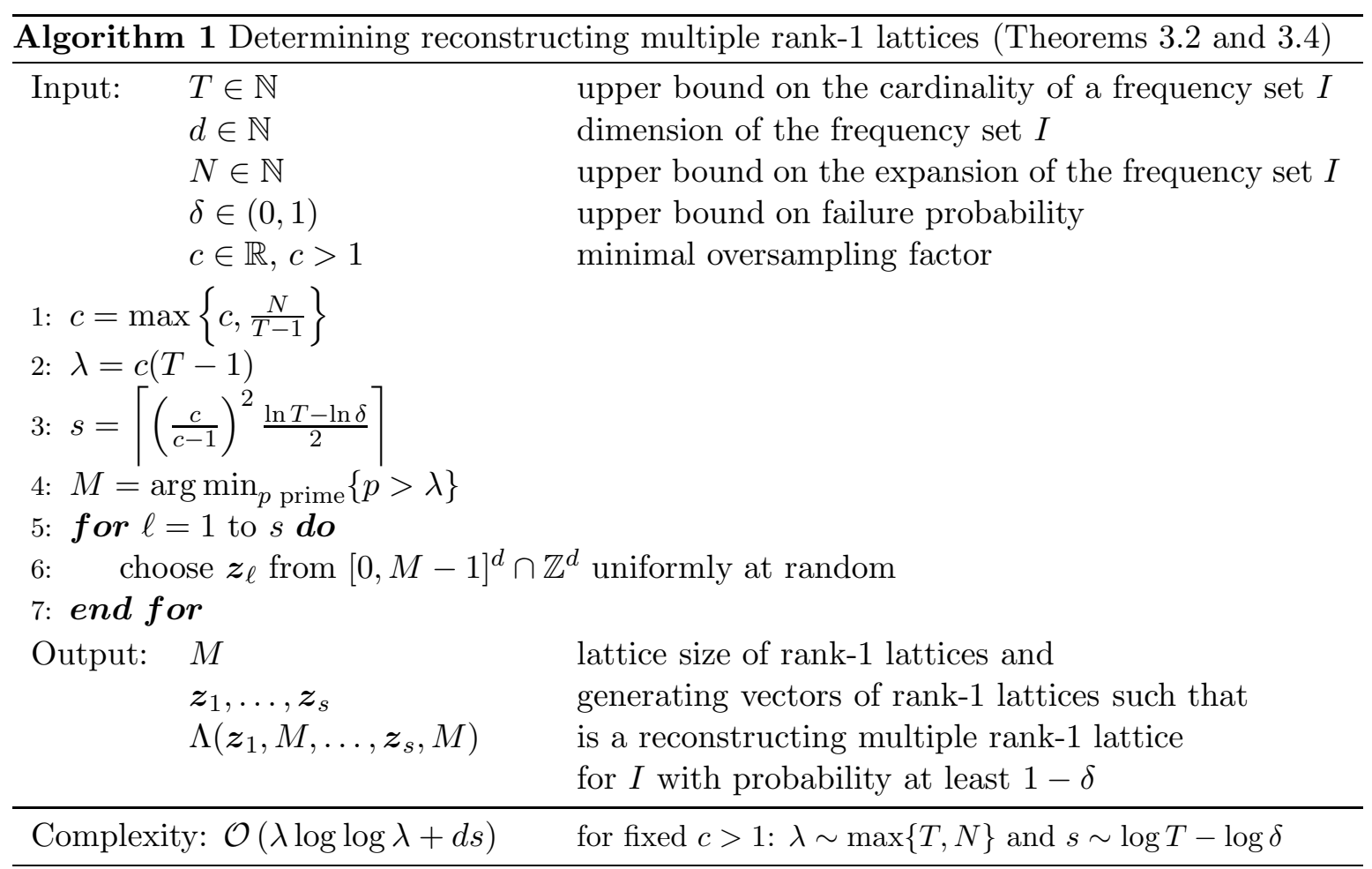

Theorem 3.4. Choosing $s, \lambda$ and $\Lambda\left(z_{\ell}, M_{\ell}\right), \ell=1, \ldots, s$, as stated in Theorem 3.2 or Corollary 3.3, the probability that the Fourier matrix $\boldsymbol{A}\left(\bigcup_{\ell=1}^{s} \Lambda\left(\boldsymbol{z}_{\ell}, M_{\ell}\right), I\right)$ has full column rank is bounded from below by $1-\delta$.

Proof. We refer to the proof of Theorem 3.2 and estimate

$$
1-\mathcal{P}\left(\bigcap_{\boldsymbol{k} \in I}\left\{\sum_{\ell=1}^{s} Y_{\ell}^{\boldsymbol{k}}<s\right\}\right)=\mathcal{P}\left(\bigcup_{\boldsymbol{k} \in I}\left\{\sum_{\ell=1}^{s} Y_{\ell}^{\boldsymbol{k}}=s\right\}\right) \leq \sum_{\boldsymbol{k} \in I} \mathcal{P}\left(\sum_{\ell=1}^{s} Y_{\ell}^{\boldsymbol{k}}=s\right) \leq T \frac{\delta}{T} .
$$

using the union bound. Let us assume that $\bigcap_{\boldsymbol{k} \in I}\left\{\sum_{\ell=1}^{s} Y_{\ell}^{\boldsymbol{k}}<s\right\}$ occurs. Then, the proof of [10, Thm 4.4] yields the full column rank of the Fourier matrix $\boldsymbol{A}\left(\bigcup_{\ell=1}^{s} \Lambda\left(\boldsymbol{z}_{\ell}, M_{\ell}\right), I\right)$.

Due to Theorem 3.4, Theorem 3.2 describes an approach that determines reconstructing sampling sets for trigonometric polynomials supported on given frequency sets $I$ with probability at least $1-\delta_{s}$, where

$$
\delta_{s}=T \mathrm{e}^{-2\left(\frac{c-1}{c}\right)^{2} s}
$$

is an upper bound on the probability that the approach fails. A slight simplification of the strategy is given as Algorithm 1, where we avoid to construct the set $P_{\lambda, 1}^{I}$. To this end, we readjust the oversampling factor $c$ in line 1 such that the set $P_{\lambda, 1}^{I}$ is forced to consist of the smallest prime larger than $\lambda$, which is contained in the interval $(\lambda, 2 \lambda]$ due to Bertrand's postulate and can be found using the sieve of Eratosthenes in $\mathcal{O}(\lambda \log \log \lambda)$. We solely use this prime number as lattice size for each of the single rank-1 lattice parts of the output of Algorithm 1, i.e., we fix $n=1$. Furthermore, Algorithm 2 uses the same strategy as Algorithm 1 with the difference that the output of Algorithm 2 is a multiple rank-1 lattice that consists 
of single rank-1 lattices with pairwise distinct lattice sizes. At this point, we would like to discuss the choice of the rank-1 lattice sizes $M_{j}$ in Algorithm 2 line 5. The theoretical considerations in Corollary 3.3 suggests to choose $s$ distinct prime numbers $M_{j}$ from $P_{\lambda, n}^{I}$ at random without replacement. Due to the readjustment of $c$, cf. line 1 , the $s$ smallest prime numbers larger than $\lambda$ are contained in $P_{\lambda, s}^{I}$, i.e., we choose $n=s$. Since we have to choose each element of $P_{\lambda, s}^{I}$ exactly once and the choices of the generating vectors do not depend on each other, the arrangement of the lattice sizes does not matter. Consequently, we can disregard the randomness in the choice of the lattice sizes $M_{1}, \ldots, M_{s}$. A simple example will demonstrate the advantages of the readjustment of $c$ in line 1 of Algorithm 1. In addition, the example treats the readjustment of $c$ in Algorithm 2 as well.

Example 3.5. The readjustment of $c$ in Algorithm 1, cf. line 1, allows for lower numbers of sampling nodes within the output of Algorithm 1. For instance, we assume $T=|I|=11$, $N_{I}=80, c=2$, and $\delta=\frac{11}{\mathrm{e}^{6}} \approx 0.0273$. Line 1 will readjust $c=8$ in that case.

We consider the above discussed approaches without using the readjustment compared to the indicated Algorithms 1 and 2 in the following table. The number $M=1-s+\sum_{r=1}^{s} M_{r}$ within the two lines right at the bottom is the number of sampling values that we achieve using the approach of distinct prime lattice sizes. The other rows show $M$, which is in fact an upper bound on the number of the sampling values within $\left|\Lambda\left(\boldsymbol{z}_{1}, M_{1}, \ldots, \boldsymbol{z}_{s}, M_{s}\right)\right|$ since we may observe $\left|\Lambda\left(\boldsymbol{z}_{j}, M_{j}\right) \cap \Lambda\left(\boldsymbol{z}_{r}, M_{r}\right)\right|>1$ for $1 \leq j<r \leq s$.

\begin{tabular}{lrrrr|rrr}
\hline & $c$ & $T$ & $N_{I}$ & $\delta$ & $s$ & $\lambda$ & upper bound on $M$ \\
\hline fixed $c, M_{r} \in P_{\lambda, 1}^{I}$ & 2 & 11 & 80 & $11 \mathrm{e}^{-6}$ & 12 & 80 & 985 \\
Algorithm 1 & 8 & 11 & 80 & $11 \mathrm{e}^{-6}$ & 4 & 80 & 329 \\
\hline \hline fixed $c$, distinct $M_{r} \in P_{\lambda, s}^{I}$ & 2 & 11 & 80 & $11 \mathrm{e}^{-6}$ & 12 & 80 & 1325 \\
Algorithm 2 & 8 & 11 & 80 & $11 \mathrm{e}^{-6}$ & 4 & 80 & 367 \\
\hline
\end{tabular}

We would like to stress the highly interesting fact, that Algorithm 1 as well as Algorithm 2 does not require the input of the frequency set $I$. The only input parameters we need are in some sense the key data of the frequency set $I$, i.e., the cardinality $T$ and the expansion $N$ of the frequency set $I$. This observation leads to the following point of view. Applying Algorithm 1 or 2 with fixed $T, N, \delta$, and $c$, leads to a multiple rank-1 lattice. Then, with probability at least $1-\delta$ this multiple rank-1 lattice is a reconstructing multiple rank-1 lattice for a frequency set $I$ with cardinality $|I| \leq T$ and upper bound $N$ on the expansion $N_{I}$. The crucial difference to the following considerations is that the frequency set $I$ itself needs not to be known.

Nevertheless, the multiple rank-1 lattices that are built using Algorithms 1 and 2 do not guarantee the reconstruction property, i.e., the full rank of the Fourier matrix $\boldsymbol{A}$, cf. (1.3), for specific, given frequency sets $I$. In general, this reconstruction property can not be checked easily. For instance, the computation of the echelon form of the matrix $\boldsymbol{A}\left(\Lambda\left(\boldsymbol{z}_{1}, M_{1}, \ldots, \boldsymbol{z}_{s}, M_{s}\right), I\right)$ or (lower bounds on) the smallest singular value of $\boldsymbol{A}\left(\Lambda\left(\boldsymbol{z}_{1}, M_{1}, \ldots, \boldsymbol{z}_{s}, M_{s}\right), I\right)$ (may) prove the reconstruction property. Corresponding test methods have a complexity in $\Omega\left(|I|^{2}\right)$, which will cause huge computational costs if the frequency set $I$ has a high cardinality. Accordingly, the direct validation of the reconstruction 


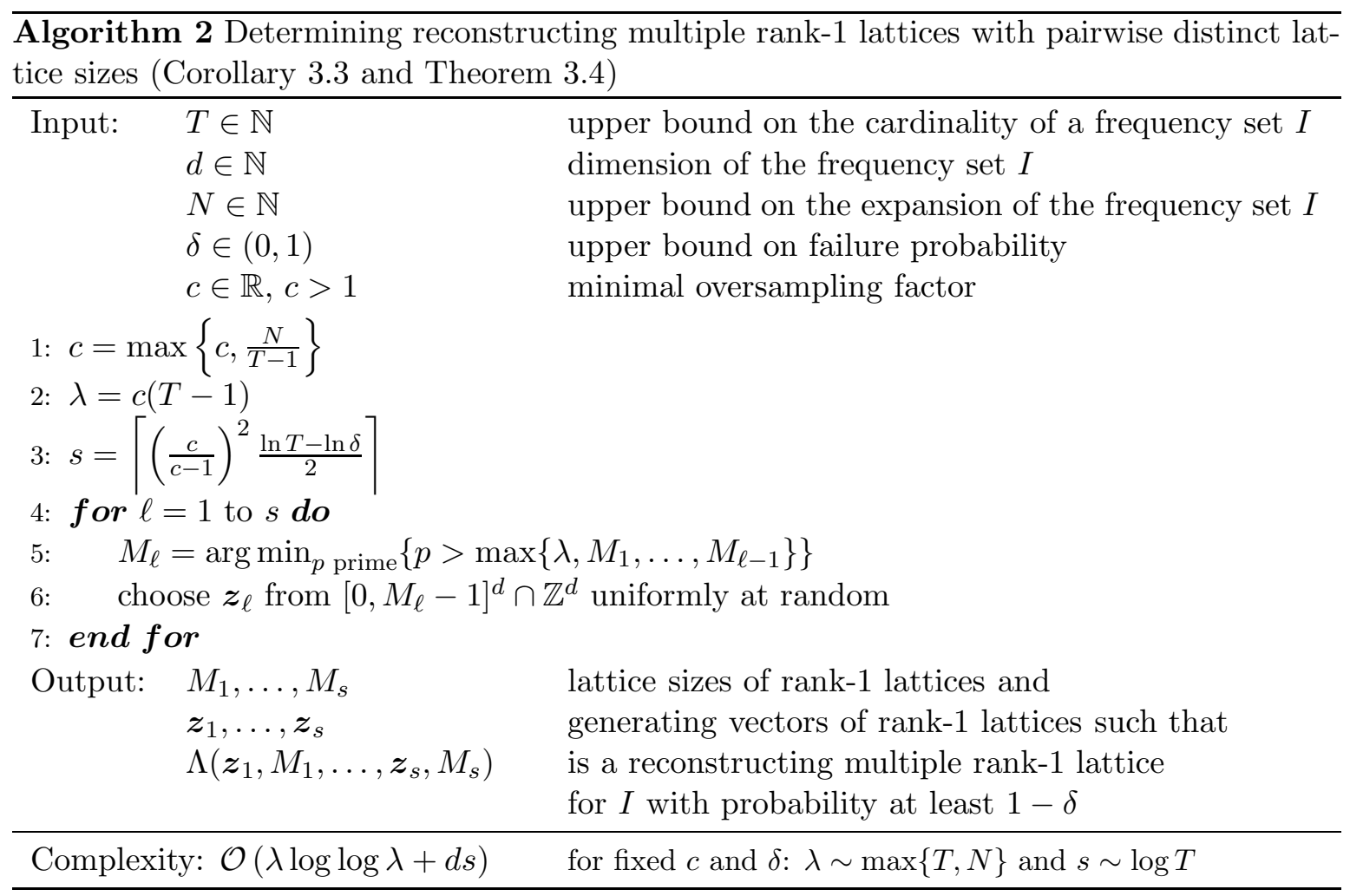

property is not convenient. For that reason, we suggest to check the conditions on the multiple rank-1 lattice $\boldsymbol{A}\left(\Lambda\left(\boldsymbol{z}_{1}, M_{1}, \ldots, \boldsymbol{z}_{s}, M_{s}\right), I\right)$ that leads to the statements in Theorems 3.2 and 3.4, i.e., we consider the sets

$$
I_{r}:=\left\{\boldsymbol{k} \in I: \boldsymbol{k} \cdot \boldsymbol{z}_{r} \not \equiv \boldsymbol{h} \cdot \boldsymbol{z}_{r} \bmod M_{r} \text { for all } \boldsymbol{h} \in I \backslash\{\boldsymbol{k}\}\right\}, \quad r=1, \ldots, s .
$$

Our theoretical considerations in the proofs of Theorem 3.4 estimated the probability that each $\boldsymbol{k} \in I$ is contained in at least one $I_{r}, r=1, \ldots, s$. In other words, we estimated the probability that the equation

$$
\bigcup_{r=1}^{s} I_{r}=I
$$

holds. The complexity of the computation of each $I_{r}$ is in $\mathcal{O}((d+\log |I|)|I|)$. The union of all $I_{r}, r=1, \ldots, s$ is also computable in $\mathcal{O}(d s|I| \log (s|I|))$, i.e., bounded by $C_{\delta} d|I| \log ^{2}|I|$, where $C_{\delta}$ depends on $\delta$ and does not depend on the cardinality $|I|$ or the dimension $d$. Accordingly, the computational costs to check (3.8) is in $\mathcal{O}\left(d|I| \log ^{2}|I|\right)$. Certainly, this strategy will not detect each reconstructing multiple rank-1 lattice, cf. Example 4.3, since equation (3.8) is sufficient but not necessary for the reconstruction property.

The last considerations give us a completely new perspective on the construction of multiple rank-1 lattices. In general, each $\boldsymbol{k} \in I$ may be contained in more than one $I_{r}, r \in\{1, \ldots, s\}$.

On the one hand, it may happen that the equation $\bigcup_{r=1}^{\tilde{s}} I_{r}=I$ with $\tilde{s}<s$ holds. In that case, even the multiple rank-1 lattice $\Lambda\left(\boldsymbol{z}_{1}, M_{1}, \ldots, \boldsymbol{z}_{\tilde{s}}, M_{\tilde{s}}\right)$ is a reconstructing rank-1 lattice for the frequency set $I$, i.e., the last $s-\tilde{s}$ rank- 1 lattices do not provide additional information 
and we can save $M_{\tilde{s}+1}+\ldots+M_{s}-s+\tilde{s}$ sampling points without loosing information about the trigonometric polynomial $p \in \Pi_{I}$.

On the other hand, it may happen that the inequality $\bigcup_{r=1}^{s} I_{r}<I$ holds since there is a significant probability that Algorithm 1 (or Algorithm 2, respectively) fails. Choosing a few additional rank-1 lattices may built a reconstructing multiple rank-1 lattice for $I$.

From this perspective, one can choose successively lattice sizes $M_{\ell}$ from $P_{\lambda, n}^{I}$ and generating vectors $\boldsymbol{z}_{\ell}$ from $\left[0, M_{\ell}-1\right]^{d}, \ell=1, \ldots$ The bound $\delta_{s}$, cf. (3.7), decreases exponentially in $s$, which implies that we can stop our strategy at a large enough $\tilde{s}$. In fact, a simple stopping criterion is easy to determine. The set

$$
\bigcup_{r=1}^{\tilde{s}}\left\{\boldsymbol{k} \in I: \boldsymbol{k} \cdot \boldsymbol{z}_{r} \not \equiv \boldsymbol{h} \cdot \boldsymbol{z}_{r} \bmod M_{r} \text { for all } \boldsymbol{h} \in I \backslash\{\boldsymbol{k}\}\right\}
$$

must be equal to the frequency set $I$.

Moreover, the equality

$$
\begin{aligned}
& \bigcup_{r=1}^{\ell-1}\left\{\boldsymbol{k} \in I: \boldsymbol{k} \cdot \boldsymbol{z}_{r} \not \equiv \boldsymbol{h} \cdot \boldsymbol{z}_{r} \bmod M_{r} \text { for all } \boldsymbol{h} \in I \backslash\{\boldsymbol{k}\}\right\} \\
& \quad=\bigcup_{r=1}^{\ell}\left\{\boldsymbol{k} \in I: \boldsymbol{k} \cdot \boldsymbol{z}_{r} \not \equiv \boldsymbol{h} \cdot \boldsymbol{z}_{r} \bmod M_{r} \text { for all } \boldsymbol{h} \in I \backslash\{\boldsymbol{k}\}\right\}
\end{aligned}
$$

yields that the additional sampling values on the sampling nodes that come from $\Lambda\left(\boldsymbol{z}_{\ell}, M_{\ell}\right)$ do not contribute to get closer to the goal, which is the equality of the frequency set $I$ and the joined frequency set in (3.9). From this point of view, we can leave out $\Lambda\left(\boldsymbol{z}_{\ell}, M_{\ell}\right)$ without loosing significant information.

We recapitulate the strategy based on Theorem 3.2 and the last-mentioned ideas in Algorithm 3 and estimate the number of sampling nodes $M=\left|\Lambda\left(\boldsymbol{z}_{1}, M_{1}, \ldots, \boldsymbol{z}_{\tilde{s}}, M_{\tilde{s}}\right)\right|$ of the outputs of Algorithm 3.

With probability not less than $1-\delta, 0<\delta<1$, Algorithm 3 determines a reconstructing multiple rank-1 lattice $\bigcup_{\ell=1}^{\tilde{s}} \Lambda\left(\boldsymbol{z}_{\ell}, M_{\ell}\right), \tilde{s} \leq s$ from (3.3), for the frequency set $I$, which consists of at most

$$
M:=\left|\Lambda\left(\boldsymbol{z}_{1}, M_{1}, \ldots, \boldsymbol{z}_{\tilde{s}}, M_{\tilde{s}}\right)\right| \leq 1-\tilde{s}+\sum_{\ell=1}^{\tilde{s}} M_{\ell} \leq s \max _{p \in P_{n}^{I, \lambda}} p
$$

sampling nodes. Since, we estimate $\tilde{s} \leq s$ in (3.10), the estimate also holds for Algorithm 1. For the same reason, the statement of the following corollary will also be valid for Algorithm 1.

Corollary 3.6. Let $0<\delta<1,1<c \in \mathbb{R}$, and $I \subset \mathbb{Z}^{d}$ a frequency set of cardinality $T=$ $|I| \geq 2, T<\infty$. We determine $\lambda$ and $s$ as defined in (3.2) and (3.3). Furthermore, we assume $N_{I} \leq \lambda:=c(T-1)$, cf. (2.3), and we fix $n=1$. Due to Bertrand's postulate and Lemma 2.5, Algorithm 3 composes a reconstructing multiple rank-1 lattice $\Lambda\left(\boldsymbol{z}_{1}, M_{1}, \ldots, \boldsymbol{z}_{\tilde{s}}, M_{\tilde{s}}\right)$ for the frequency set $I$ that consists of at most

$$
M \leq 1-\tilde{s}+\sum_{\ell=1}^{\tilde{s}} M_{\ell} \leq\left\lceil\left(\frac{c}{c-1}\right)^{2} \frac{\ln T-\ln \delta}{2}\right\rceil 2 c(T-1)<C_{c, \delta} T \ln T
$$




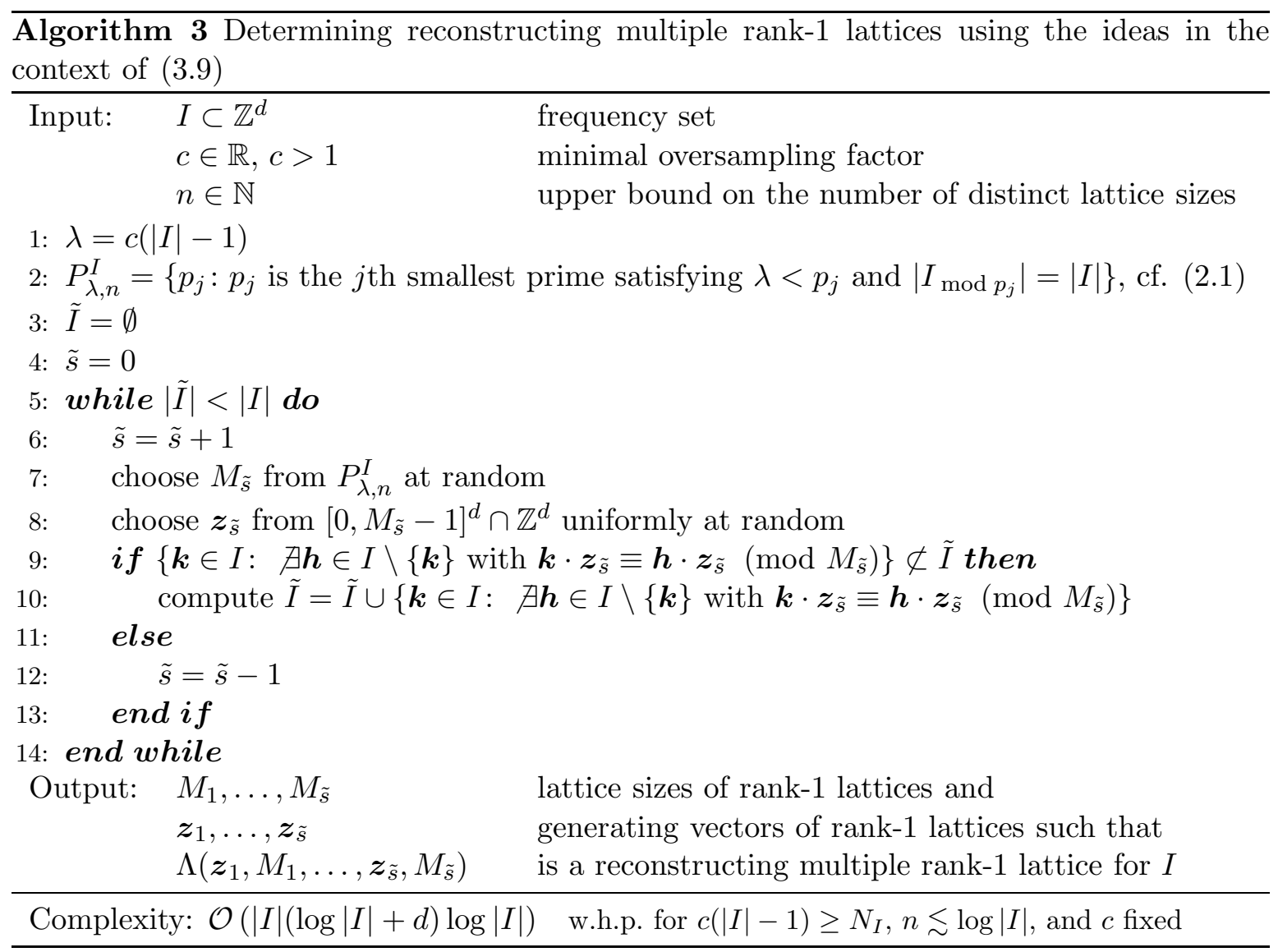

sampling nodes with probability $1-\delta$. For the sake of completeness, we give a simple upper bound on

$$
C_{c, \delta} \leq 2 c\left(\left(\frac{c}{c-1}\right)^{2} \frac{1-\log _{2} \delta}{2}+\log _{2} \mathrm{e}\right),
$$

that depends logarithmically on the upper bound $\delta$ of the failure probability.

Not surprisingly, the reconstructing multiple rank-1 lattices that are constructed using Algorithm 3 need some oversampling. However, the occurring oversampling factors $\frac{M}{T}$ are bounded by a term $C_{c, \delta} \ln T$ with probability $1-\delta$ in the case that the expansion of the frequency set $I$ is bounded by $N_{I} \leq c(T-1)$. Consequently, the oversampling factor scales at most logarithmically in $T$ and is independent on the dimension $d$ of the frequency set $I$.

As mentioned above, we will also follow the approach of multiple rank-1 lattices as subsampling schemes of huge single rank-1 lattices. To this end, we will construct reconstructing multiple rank-1 lattices that consists of rank-1 lattices which have pairwise distinct lattice sizes $M_{1}, \ldots, M_{\tilde{s}}$, cf. Algorithm 4. Due to Corollary 3.3 and Theorem 3.4 the estimate

$$
M:=\left|\Lambda\left(\boldsymbol{z}_{1}, M_{1}, \ldots, \boldsymbol{z}_{\tilde{s}}, M_{\tilde{s}}\right)\right| \leq \tilde{s} \max _{p \in P_{\tilde{s}}^{I, \lambda}} p \leq s \max _{p \in P_{s}^{I, \lambda}} p
$$

hold for $2 \leq|I|=T<\infty, 1>\delta>0,1<c \in \mathbb{R}, \lambda$ and $s$ as determined in (3.2) and (3.3) with probability $1-\delta$. In order to estimate the number of sampling nodes of the outputs of Algorithm 4, we need an upper bound on the largest prime within $P_{\lambda, s}^{I}$. Taking into account 
some additional requirements on the number $\lambda$, the inclusion $P_{\lambda, s}^{I} \subset(\lambda, 2 \lambda]$ holds. A similar strategy is already applied in [1, L.9].

Since we use the number $s$ as upper bound on $\tilde{s}$ in (3.12), the estimate of the following corollary hold for Algorithm 4 and for Algorithm 2 as well.

Corollary 3.7. We consider an arbitrary frequency set $I, 2 \leq|I|=T<\infty$, with expansion $N_{I}$, cf. (2.3). Furthermore, we fix $0<\delta<1,1<c \in \mathbb{R}$, and determine $s=\left\lceil\left(\frac{c}{c-1}\right)^{2} \frac{\ln T-\ln \delta}{2}\right\rceil$ and $\lambda=\max \left\{c(T-1), N_{I}, 4 s \ln s\right\}$. Then, with probability not less than $1-\delta$, Algorithm 4 determines a reconstructing multiple rank-1 lattice $\bigcup_{\ell=1}^{\tilde{s}} \Lambda\left(\boldsymbol{z}_{\ell}, M_{\ell}\right)$ for the frequency set $I$, which consists of at most

$$
M \leq 2\left\lceil\left(\frac{c}{c-1}\right)^{2} \frac{\ln T-\ln \delta}{2}\right\rceil \max \left\{c(T-1), N_{I}, 4 s \ln s\right\}
$$

sampling nodes. If $c(T-1)$ is the dominating term in the maximum in (3.13), we estimate

$$
M \leq\left\lceil\left(\frac{c}{c-1}\right)^{2} \frac{\ln T-\ln \delta}{2}\right\rceil 2 c(T-1)<C_{c, \delta} T \ln T .
$$

Proof. Since the statement in (3.12) is a direct consequence of Theorem 3.4, the goal is to prove the embedding $P_{s}^{I, \lambda} \subset(\lambda, 2 \lambda]$ which yields (3.13).

Due to the fact that $\lambda \geq N_{I}$ holds, the set $P^{I}$ contains all primes within $(\lambda, 2 \lambda]$, cf. Lemma 2.5. Thus, we have to ensure, that the interval $(\lambda, 2 \lambda]$ contains at least $s$ different prime numbers $p_{j}, j=1, \ldots, s$. We follow the argumentation in the proof of $[1$, L. 9] and distinguish four different cases:

$s \geq 4$. We have $\lambda \geq 4 s \ln s>22$. Due to [18, Cor. 3], the number of primes within the interval $\overline{(\lambda, 2 \lambda]}$ is bounded from below by $\frac{3 \lambda}{5 \ln \lambda}$ for all $\lambda \geq 20.5$. We consider the mapping $\lambda \mapsto \frac{3 \lambda}{5 \ln \lambda}$ for $\lambda>22$ and observe that this mapping monotonically increases with $\lambda$. We estimate

$$
\frac{3 \lambda}{5 \ln \lambda} \geq s \frac{\ln s^{12 / 5}}{\ln (4 s \ln s)} \geq s
$$

for $s^{12 / 5} \geq 4 s \ln s$, which is fulfilled for $s \geq 4$.

$\underline{s=3}$. We determine $\lambda \geq 12 \ln 3>13$ and know that for $\lambda>22$ there exist at least 4 prime numbers within the interval $(\lambda, 2 \lambda]$. The same interval for $13<\lambda \leq 22$ should contain at least 3 prime numbers. We determine

$$
\begin{array}{llll}
\{17,19,23\} \subset(\lambda, 2 \lambda] & \text { for } & \lambda \in[11.5,17) \\
\{23,29,31\} \subset(\lambda, 2 \lambda] & \text { for } & \lambda \in[17,22]
\end{array}
$$

Hence, for all $\lambda \geq 11.5$ the interval $(\lambda, 2 \lambda]$ contains at least 3 different prime number.

$\underline{s=2}$. We determine $\lambda \geq 8 \ln 2>5.5$. With the argumentations above, we determine two prime numbers within the sets $(\lambda, 2 \lambda]$.

$$
\begin{aligned}
& \{7,11\} \subset(\lambda, 2 \lambda] \quad \text { for } \quad \lambda \in[5.5,7) \\
& \{11,13\} \subset(\lambda, 2 \lambda] \quad \text { for } \quad \lambda \in[7,11) \\
& \{17,19\} \subset(\lambda, 2 \lambda] \quad \text { for } \quad \lambda \in[11,17)
\end{aligned}
$$


For larger $\lambda$ we have at least 3 prime numbers in the interval under consideration.

$\underline{s=1}$. We determine $\lambda \geq c(T-1)>1$. Due to Bertrand's postulate, there exists at least one prime in the interval $(\lambda, 2 \lambda]$.

The term $4 s \ln s$ within the termination of $\lambda$ ensures the existence of sufficiently many prime numbers that are bounded from above by $2 \lambda$ as well as large enough, i.e., larger than $\lambda$. According to Corollary 3.3, Algorithm 4 determines a multiple rank-1 lattice of pairwise distinct prime sizes less or equal to $2 \lambda$ with probability at least $1-\delta$. We call $M$ the total number of sampling nodes within the output of Algorithm 4 and observe the estimates in (3.13) and (3.14).

The last corollary provides a specific estimate of the number of sampling nodes within a reconstructing multiple rank-1 lattice that is determined by Algorithm 4. We notice that the statement is almost the same as in Corollary 3.6. The single difference is that an estimate $M \leq 2 s c(T-1) \leq C_{c, \delta} T \ln T$ requires $\lambda:=c(T-1) \geq 4 s \ln s$ in addition to $\lambda:=c(T-1) \geq N_{I}$. A rough estimate yields $s \ln s \leq s^{2} \in \mathcal{O}\left(\log ^{2} T\right)$ for fixed $c$ and $\delta$. Thus, a large enough $T$ implies the inequality $c(T-1) \geq 4 s \ln s$. Assuming (3.14) is fulfilled, the sampling sets that are determined using Algorithm 4 need oversampling, where the oversampling factor scales logarithmically in terms of the cardinality $T$ of the frequency set $I$.

At this point, we would like to stress on the modifications of Algorithm 4. We deterministically choose the lattice sizes $M_{\ell}$ in Line 8 . The theoretical results are based on the idea that we choose $s$ rank-1 lattices of distinct sizes at random, where the lattice sizes are contained in the set $P_{\lambda, s}^{I}$. Since we have to choose each element of $P_{\lambda, s}^{I}$ exactly once and the choices of the generating vectors do not depend on each other, the arrangement of the lattice sizes does not matter. Moreover, the termination criterion of Algorithm 4 is the one that we used in Algorithm 3, which is already discussed above, cf. the context of (3.9). This termination criterion may cause that the number $\tilde{s}$ of rank-1 lattices that are determined by Algorithm 4 is much less than the number $s$, cf. (3.3), that is theoretically determined. Taking this into account, the growing sequence of lattice sizes $M_{1}, \ldots, M_{\tilde{s}}$ may avoid the largest possible lattice sizes.

Another point to mention is that Algorithm 4 constructs a reconstructing multiple rank-1 lattice only with high probability, provided that $\delta$ is small. Possibly, the output of Algorithm 4 is not a reconstructing multiple rank-1 lattice for the frequency set $I$. In detail, if $\tilde{s}=s$ and $\tilde{I} \subsetneq I$ hold, Algorithm 4 is failing. Clearly, one can simply detect these cases and may avoid the corrupt output by restarting Algorithm 4 or increasing $s$, where the latter approach leads to multiple rank-1 lattices with a number of sampling nodes that may not allow for the estimate in (3.13).

Anyway, a simple example deals with the result of Corollary 3.7 .

Example 3.8. We fix $c=3$ and $\delta=\frac{1}{100}$. For a frequency set $I \subset \mathbb{Z}^{d}$ with $T:=|I| \geq 64$ and $N_{I} \leq 3(T-1)$, we apply Algorithm 4 in order to construct a reconstructing multiple rank-1 lattice $\Lambda\left(\boldsymbol{z}_{1}, M_{1}, \ldots, \boldsymbol{z}_{\ell}, M_{\ell}\right)$ for $I$. Then Algorithm 4 will succeed with a total number of sampling values $M=1-s+\sum_{j=1}^{\ell} M_{\ell}<16 T \ln T$ with probability greater than $1-\delta=0.99$. 


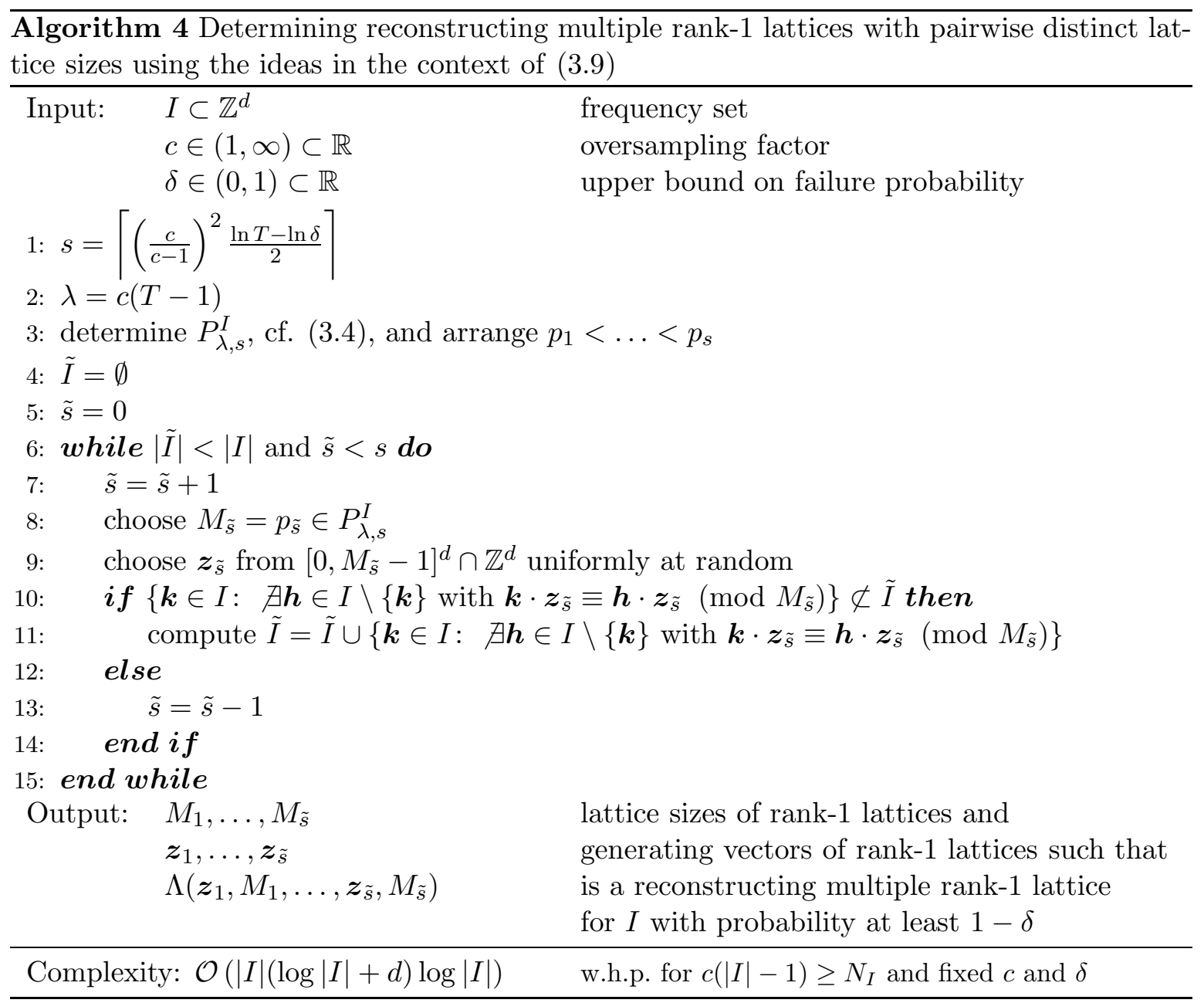

Proof. We estimate

$$
\begin{aligned}
s & \leq\left(\frac{c}{c-1}\right)^{2} \frac{\ln T-\ln \delta}{2}+1=\frac{9}{4} \frac{\ln T+\ln 100}{2}+1<\frac{9}{4} \frac{\ln T+\frac{10}{9} \ln 64}{2}+1 \\
& <\frac{19}{8} \ln T+1<\frac{21}{8} \ln T \\
4 s \ln s & \leq 3(T-1)
\end{aligned}
$$

for $T \geq 64$. Consequently, the equality $\lambda=3(T-1)$ in Cor. 3.7 holds. The number $M$ of used sampling values is bounded by

$$
M \leq 2 \lambda s \leq 6(T-1) \frac{21}{8} \ln T<\frac{63}{4} T \ln T .
$$

\section{Heuristic upgrades}

In this Section, we consider Theorem 3.4 in more detail and iteratively apply the ideas therein. 


\subsection{Iteratively determined lattices}

We denote $I_{1}=I, T_{1}=T, \lambda_{1}=\lambda$ and fix the number $n=1$ in Theorem 3.2, i.e., the set $P_{\lambda_{1}, 1}^{I_{1}}$ contains only one prime number $M_{1}$ larger than $\lambda_{1}$. If the strategies described in Section 3 succeeds, each Fourier coefficient of a specific frequency within $I_{1}$ can be uniquely reconstructed using one of the $s$ rank-1 lattices $\Lambda\left(\boldsymbol{v}_{\ell}, M_{1}\right), \ell=1, \ldots, s$, that are determined according to Theorem 3.2, cf. Theorem 3.4 and its interpretation in the context of (3.8). This yields

$$
I_{1}=\bigcup_{\ell=1}^{s}\left\{\boldsymbol{k} \in I_{1}: \boldsymbol{k} \cdot \boldsymbol{v}_{\ell} \not \equiv \boldsymbol{h} \cdot \boldsymbol{v}_{\ell}\left(\bmod M_{1}\right), \forall \boldsymbol{h} \in I_{1} \backslash\{\boldsymbol{k}\}\right\}
$$

and we estimate

$$
\begin{aligned}
T & \leq \sum_{\ell=1}^{s}\left|\left\{\boldsymbol{k} \in I_{1}: \boldsymbol{k} \cdot \boldsymbol{v}_{\ell} \not \equiv \boldsymbol{h} \cdot \boldsymbol{v}_{\ell}\left(\bmod M_{1}\right), \forall \boldsymbol{h} \in I_{1} \backslash\{\boldsymbol{k}\}\right\}\right| \\
\frac{T}{s} & \leq \max _{\ell=1, \ldots, s}\left|\left\{\boldsymbol{k} \in I_{1}: \boldsymbol{k} \cdot \boldsymbol{v}_{\ell} \not \equiv \boldsymbol{h} \cdot \boldsymbol{v}_{\ell}\left(\bmod M_{1}\right), \forall \boldsymbol{h} \in I_{1} \backslash\{\boldsymbol{k}\}\right\}\right| .
\end{aligned}
$$

Accordingly, with probability at least $1-\delta$ we can choose $\ell^{\prime} \in\{1, \ldots, s\}$, determine $\boldsymbol{z}_{1}=\boldsymbol{v}_{\ell^{\prime}}$ such that sampling along the rank-1 lattice $\Lambda\left(z_{1}, M_{1}\right)$ allows for the unique reconstruction of at least $\frac{T}{s}$ Fourier coefficients of each polynomial $p_{1}=\sum_{\boldsymbol{k} \in I_{1}} \hat{p}_{\boldsymbol{k}} \mathrm{e}^{2 \pi \mathrm{i} \boldsymbol{k} \cdot \circ} \in \Pi_{I_{1}}$. Let $\Lambda\left(\boldsymbol{z}_{1}, M_{1}\right)$ be a rank-1 lattice coming from the ideas above. We define

$$
\tilde{I}_{1}=\left\{\boldsymbol{k} \in I_{1}: \boldsymbol{k} \cdot \boldsymbol{z}_{1} \not \equiv \boldsymbol{h} \cdot \boldsymbol{z}_{1} \bmod M_{1}, \forall \boldsymbol{h} \in I_{1} \backslash\{\boldsymbol{k}\}\right\},
$$

where we assume that $\Lambda\left(\boldsymbol{z}_{1}, M_{1}\right)$ is a rank-1 lattice such that $\left|\tilde{I}_{1}\right| \geq \frac{T}{s}$ holds. We determine the Fourier coefficients of $p_{1}$

$$
\hat{p}_{\boldsymbol{k}}=M_{1}^{-1} \sum_{l=0}^{M_{1}-1} p_{1}\left(\frac{j}{M_{1}} \boldsymbol{z}_{1}\right) \mathrm{e}^{-2 \pi \mathrm{i} \frac{l}{M_{1}} \boldsymbol{k} \cdot \boldsymbol{z}_{1}}
$$

for all frequencies $\boldsymbol{k} \in \tilde{I}_{1}$ using a single one-dimensional FFT. The essential steps are the computation of the frequency set $\tilde{I}_{1}$ and a rank-1 lattice FFT, cf. [9, Alg. 3.2], which have a complexity in $\mathcal{O}\left(\left(d+\log T_{1}\right) T_{1}\right)$ and $\mathcal{O}\left(M_{1} \log M_{1}+d T_{1}\right)$, respectively. Therefore the total complexity of this approach is in $\mathcal{O}\left(M_{1} \log M_{1}+\left(d+\log T_{1}\right) T_{1}\right)$. In a next step we consider the polynomial

$$
p_{2}(\boldsymbol{x})=p_{1}(\boldsymbol{x})-\sum_{\boldsymbol{k} \in \tilde{I}_{1}} \hat{p}_{\boldsymbol{k}} \mathrm{e}^{2 \pi \mathrm{i} \boldsymbol{k} \cdot \boldsymbol{x}}=\sum_{\boldsymbol{k} \in I_{1} \backslash \tilde{I}_{1}} \hat{p}_{\boldsymbol{k}} \mathrm{e}^{2 \pi \mathrm{i} \boldsymbol{k} \cdot \boldsymbol{x}}
$$

and reduce the problem to the reconstruction of the trigonometric polynomial $p_{2} \in \Pi_{I_{2}}$ with a frequency set $I_{2}=I_{1} \backslash \tilde{I}_{1}$ of lower cardinality. We apply Theorems 3.2 with $n=1$ and 3.4 to the trigonometric polynomial $p_{2}$ in (4.2). In this way we apply this strategy successively until the frequency set $I_{j}=\emptyset$ is empty. Algorithm 5 depicts the construction of the sampling sets that are formed using this strategy. A detailed analysis of the algorithm leads to the following findings. 


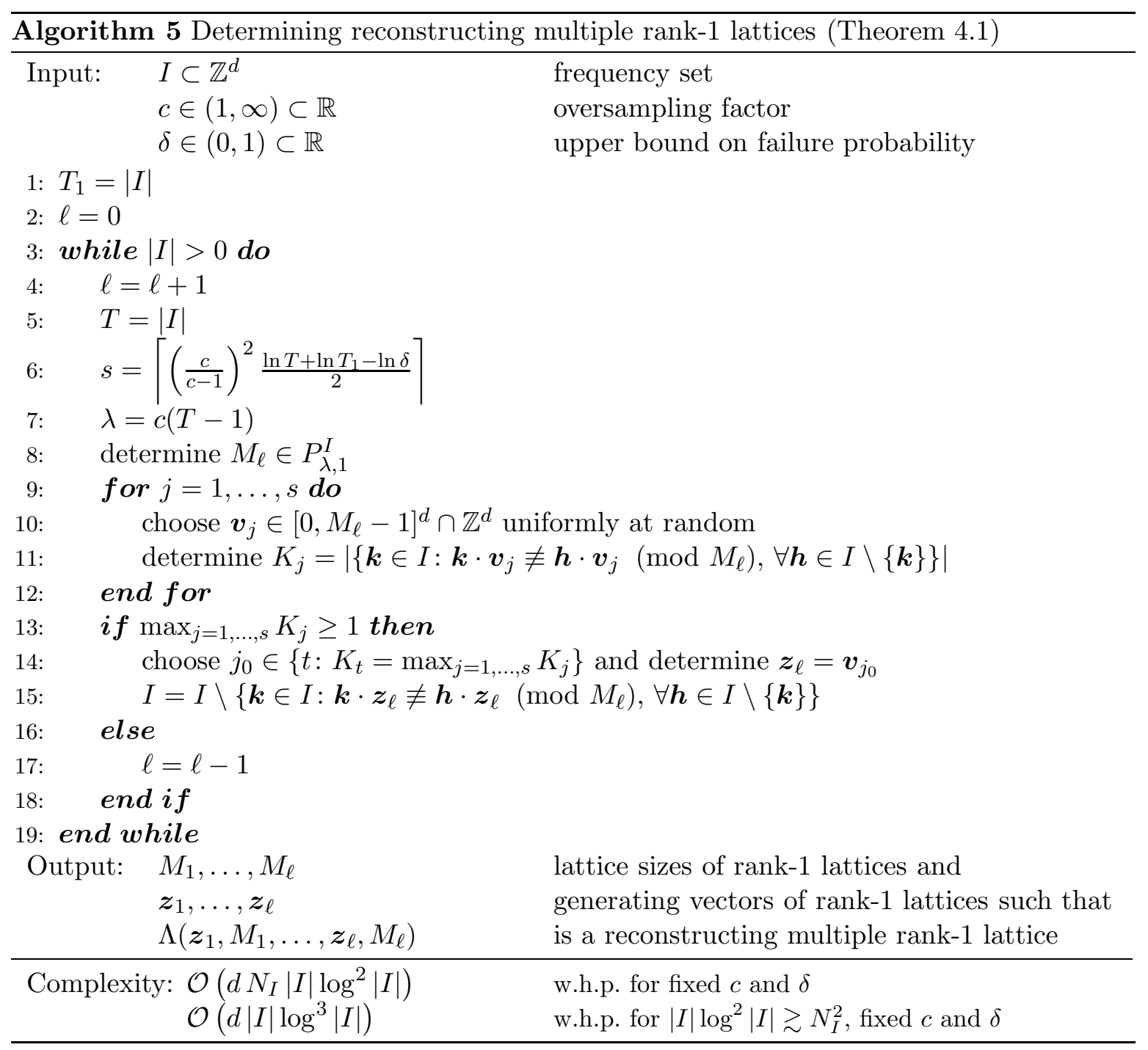

Theorem 4.1. We assume $I_{1}:=I \subset \mathbb{Z}^{d}$ is a frequency set of finite cardinality $\left|I_{1}\right|<\infty$, $\delta \in \mathbb{R}, 0<\delta<1$, and $c \in \mathbb{R}, c>1$ fixed real numbers. In addition, we denote for $j=1, \ldots$

- $T_{j}:=\left|I_{j}\right|$,

- $\lambda_{j}:=c\left(T_{j}-1\right)$,

- $s_{j}:=\left\lceil\left(\frac{c}{c-1}\right)^{2} \frac{\ln T_{j}+\ln T_{1}-\ln \delta}{2}\right\rceil$,

- $M_{j} \in P_{\lambda_{j}, 1}^{I_{j}}$ prime number larger than $\lambda_{j}$,

- $\boldsymbol{z}_{j}$ the best possible of $s_{j}$ chosen random generating vectors,

- $I_{j+1}:=\left\{\boldsymbol{k} \in I_{j}: \exists \boldsymbol{h} \in I_{j} \backslash\{\boldsymbol{k}\}\right.$ s.t. $\left.\boldsymbol{k} \cdot \boldsymbol{z}_{j} \equiv \boldsymbol{h} \cdot \boldsymbol{z}_{j}\left(\bmod M_{j}\right)\right\}$,

and a number $k:=\min \left\{\left\lceil s_{1} \ln T_{1}\right\rceil, T_{1}\right\}$. Then, with probability not less than $1-\frac{k \delta}{T_{1}} \geq 1-\delta$ the cardinality of the frequency set $I_{k+1}$ is zero, i.e., $\Lambda\left(\boldsymbol{z}_{1}, M_{1}, \ldots, \boldsymbol{z}_{k}, M_{k}\right)$ is a reconstructing multiple rank-1 lattice for $I$. 
Proof. We start with the frequency set $I_{1}$ and choose $s_{1}$ vectors from $\left[0, M_{1}-1\right]^{d}$ at random. Due to Theorem 3.4, Theorem 3.2, and the considerations in the context of (4.1), the best possible of the $s_{1}$ randomly chosen vectors provides $T_{2} \leq\left(1-1 / s_{1}\right) T_{1}$ with probability of at least $1-\frac{\delta}{T_{1}}$, i.e., the probability of failing this property is not greater than $\frac{\delta}{T_{1}}$.

Similar considerations can be done for $I_{j}, j=2, \ldots, k$. We have $s_{j}=\left\lceil\left(\frac{c}{c-1}\right)^{2} \frac{\ln T_{j}-\ln \frac{\delta}{T_{1}}}{2}\right\rceil$. We apply Theorems 3.4 and 3.2 on $I_{j}$ and we determine the best of $s_{j}$ randomly chosen vectors. With probability less or equal $\frac{\delta}{T_{1}}$ we observe $T_{j+1}>\left(1-1 / s_{j}\right) T_{j}$. We estimate

$$
\begin{aligned}
\mathcal{P}\left(\bigcap_{j=1}^{k}\left\{T_{j+1} \leq\left(1-1 / s_{j}\right) T_{j}\right\}\right) & =1-\mathcal{P}\left(\bigcup_{j=1}^{k}\left\{T_{j+1}>\left(1-1 / s_{j}\right) T_{j}\right\}\right) \\
& \geq 1-\sum_{j=1}^{k} \mathcal{P}\left(\left\{T_{j+1}>\left(1-1 / s_{j}\right) T_{j}\right\}\right) \geq 1-\frac{k \delta}{T_{1}}
\end{aligned}
$$

Now, we estimate the number $k$. On the one hand, if $T_{j+1} \leq\left(1-1 / s_{j}\right) T_{j}, j=1 \ldots, k$, hold, we have $T_{k+1} \leq T_{1} \prod_{j=1}^{k}\left(1-1 / s_{j}\right) \leq\left(1-1 / s_{1}\right)^{k} T_{1}$ and in particular $T_{k+1}=0$ for $\left(1-1 / s_{1}\right)^{k}<T_{1}^{-1}$, which is fulfilled for

$$
k \geq s_{1} \ln T_{1}>\frac{\ln T_{1}}{\ln s_{1}-\ln \left(s_{1}-1\right)} .
$$

On the other hand, since $\infty>s_{j} \geq 1$, the conditions $T_{j+1} \leq\left(1-1 / s_{j}\right) T_{j}, j=1 \ldots, k$, imply

$$
0 \leq T_{j+1} \leq \begin{cases}T_{j}-1 & : T_{j} \geq 1 \\ 0 & : T_{j}=0\end{cases}
$$

and $T_{T_{1}+1}=0$, i.e., even if the estimate in (4.3) yields $k \geq s_{1} \ln T_{1}$ the number $k$ of used lattices may be already bounded from above by $k \leq T_{1}$.

In summary, with probability greater than $1-\frac{k \delta}{T_{1}} \geq 1-\delta$, we construct a reconstructing multiple rank-1 lattice consisting of at most $k=\min \left\{\left\lceil s_{1} \ln T_{1}\right\rceil, T_{1}\right\}$ joined rank-1 lattices.

The latter approach is indicated in Algorithm 5. An additional restriction on the expansion $N_{I}$ of the considered frequency set $I$ allows for a rough estimate of the number $M$ of sampling nodes of the reconstructing rank-1 lattice that is constructed by Algorithm 5 .

Corollary 4.2. We assume $I_{1}:=I \subset \mathbb{Z}^{d}$ is a frequency set of finite cardinality $T_{1}=\left|I_{1}\right| \geq 3$, $\delta \in \mathbb{R}, 0<\delta<1$, and $c \in \mathbb{R}, c>1$ fixed real numbers. In addition, we assume $N_{I_{1}} \leq \frac{c\left(T_{1}-1\right)}{\ln T_{1}}$. Then, with probability at least $1-\delta$ Algorithm 5 constructs a reconstructing multiple rank-1 lattice $\Lambda\left(\boldsymbol{z}_{1}, M_{1}, \ldots, \boldsymbol{z}_{\ell}, M_{\ell}\right)$ of

$$
M \leq 1-\ell+\sum_{j=1}^{\ell} M_{j} \leq\left(2\left(\frac{c}{c-1}\right)^{2}\left(2 \ln T_{1}-\ln \delta\right)+6\right) c T_{1}
$$

sampling nodes. 
Proof. Due to Bertrand's postulate and Lemma 2.4 we can find $M_{j}, j=1, \ldots, k$, in Theorem 4.1 such that $M_{j} \leq 2 \max \left\{\lambda_{j}, N_{I_{j}}\right\} \leq 2 \max \left\{\lambda_{j}, N_{I_{1}}\right\}$. Moreover, we know that the inequality $\ell \leq k$ holds with probability at least $1-\delta$. Accordingly, we estimate

$$
\begin{aligned}
M & \leq 1-\ell+\sum_{j=0}^{\ell} M_{j} \leq 2 \sum_{j=1}^{k} \max \left\{\lambda_{j}, N_{I_{1}}\right\} \leq 2\left(k N_{I_{1}}+\sum_{j=1}^{k} \lambda_{j}\right) \\
& \leq 2 k N_{I_{1}}+2 c \sum_{j=1}^{k}\left(T_{j}-1\right) \leq 2 k \frac{c\left(T_{1}-1\right)}{\ln T_{1}}+2 c\left(-k+\sum_{j=1}^{k} T_{j}\right) \\
& \leq 2 c k\left(\frac{T_{1}-1}{\ln T_{1}}-1\right)+2 c T_{1}\left(1+\sum_{j=1}^{k-1} \prod_{l=1}^{j}\left(1-1 / s_{l}\right)\right) \\
& \leq 2 c\left(\left(s_{1}+\frac{1}{\ln T_{1}}\right) T_{1}\right)+2 c T_{1} \sum_{j=0}^{\infty}\left(1-1 / s_{1}\right)^{j} \\
& <2 c T_{1}\left(2 s_{1}+\frac{1}{\ln T_{1}}\right)^{T_{1}>\mathrm{e}} 2\left(2 s_{1}+1\right) c T_{1} \leq\left(2\left(\frac{c}{c-1}\right)^{2}\left(2 \ln T_{1}-\ln \delta\right)+6\right) c T_{1}
\end{aligned}
$$

with probability at least $1-\delta$.

We stress on the fact that the upper bound on the sampling nodes of a reconstructing multiple rank-1 lattice in (4.4) is larger than the bound in (3.11). Nevertheless the bounds are in the same order $\Theta\left(T_{1} \ln T_{1}\right)$ and $\Theta(\ln \delta)$ with respect to $T_{1}=\left|I_{1}\right|$ and $\delta$, respectively. Moreover, both upper bounds do not depend on the dimension $d$.

At this point, we comment on some characteristics of Algorithm 5. Against the theoretical considerations, we added the lines 16-18 in Algorithm 5, that avoid to use a rank-1 lattice that does not yield additional information compared to the already determined rank-1 lattices. Furthermore, Algorithm 5 will not terminate until a reconstructing rank-1 lattice for the input is determined. Assuming Algorithm 5 will not terminate implies that at one $\ell$ the while loop is an endless loop, which means that $|I|$ does not decrease. The lattice size $M_{\ell}$ is fixed in this endless loop. Accordingly, we test $n s, n=1, \ldots$, vectors $\boldsymbol{v}_{j}$ for their reconstruction property. Taking Theorem 3.2 and the estimates in (3.6) into account, we observe that the probability that a Fourier coefficient of a single fixed frequency within $I$ can not be reconstructed using $n s$ randomly chosen vectors is at most $\mathrm{e}^{-2 n s\left(\frac{c-1}{c}\right)^{2}}$, which decays exponentially in $n$. Thus, in practice we will not observe endless loops in Algorithm 5 and, consequently, the algorithm terminates. Nevertheless, the output of Algorithm 5 may not fulfill the upper bound in (4.4). From this point of view, each multiple rank-1 lattice $\Lambda\left(z_{1}, M_{1}, \ldots, \boldsymbol{z}_{\ell}, M_{\ell}\right)$ which is the output of Algorithm 5 is actually a reconstucting multiple rank-1 lattice for the frequency set $I$ under consideration. Consequently, the parameter $\delta$, the so-called upper bound on the failure probability, does not bound the probability that the reconstruction property of the sampling set $\Lambda\left(\boldsymbol{z}_{1}, M_{1}, \ldots, \boldsymbol{z}_{\ell}, M_{\ell}\right)$ fails. However, $\delta \in(0,1)$ is an upper bound on the probability that the number $\ell$ of joined rank-1 lattices is greater than $k$ in Theorem 4.1 and that the number of sampling nodes within $\Lambda\left(\boldsymbol{z}_{1}, M_{1}, \ldots, \boldsymbol{z}_{\ell}, M_{\ell}\right)$ does not fulfill inequality (4.4) in Corollary 4.2.

Furthermore, we would like to mention that the rank-1 lattice sizes $M_{1}, \ldots, M_{\ell}$, that are determined using Algorithm 5, are not necessarily distinct, and thus, the determined recon- 


\begin{tabular}{lll}
\hline Algorithm 6 Determining reconstructing & MR1L (Theorem 4.1) \\
\hline Input: & $I \subset \mathbb{Z}^{d}$ & frequency set \\
& $c \in(1, \infty) \subset \mathbb{R}$ & oversampling factor \\
$\delta \in(0,1) \subset \mathbb{R}$ & upper bound on failure probability \\
lines 1 to 7 identical with those of Algorithm 5 \\
8: determine $M_{\ell}=\min \left\{P_{\ell}^{I, \lambda} \backslash\left\{M_{1}, \ldots, M_{\ell-1}\right\}\right\}$ \\
lines 9 to 19 identical with those of Algorithm 5 \\
Output: $\quad M_{1}, \ldots, M_{\ell}$ & lattice sizes of rank-1 lattices and \\
$\boldsymbol{z}_{1}, \ldots, \boldsymbol{z}_{\ell}$ & generating vectors of rank-1 lattices such that \\
$\Lambda\left(\boldsymbol{z}_{1}, M_{1}, \ldots, \boldsymbol{z}_{\ell}, M_{\ell}\right)$ & is a reconstructing multiple rank-1 lattice \\
\hline Complexity: $\mathcal{O}\left(d N_{I}|I| \log ^{2}|I|\right)$ & w.h.p. for fixed $c$ and $\delta$ \\
$\mathcal{O}\left(d|I| \log ^{3}|I|\right)$ & w.h.p. for $|I| \log ^{2}|I| \gtrsim N_{I}^{2}$, fixed $c$ and $\delta$ \\
\hline
\end{tabular}

structing multiple rank-1 lattice is not necessarily a subsampling scheme of a huge single rank-1 lattice, cf. the context of Corollary 3.3. A simple modification on line 8 of Algorithm 5 provides even this nice property, cf. Algorithm 6. Certainly, an additional assumption $\lambda_{1}=c\left(T_{1}-1\right) \geq 4 k \ln k$ in Corollary 4.2 would guarantee the existence of at least $k$ distinct primes within the interval $\left(\lambda_{1}, 2 \lambda_{1}\right]$, cf. Corollary 3.7 for details. For $N_{I}$ small enough, an obvious estimate yields $\sum_{j=1}^{\ell} M_{j} \leq 2 k c T_{1} \in \mathcal{O}\left(T_{1} \ln ^{2} T_{1}\right)$, i.e., in comparison to the statement of Corollary 4.2 the estimate suffers from an additional logarithmic term in $T_{1}$. However, we did not exploit the decreasing $\lambda_{j}, j=1, \ldots, k$, in the obvious estimate. Depending on $T_{1}$, $N_{I_{1}}, \delta$, and $c$, a detailed analysis of this estimate might save a logarithmic term here.

\subsection{Stretching the set $P^{I}$}

Up to now, all presented algorithms need to choose the lattice sizes from a set $P_{\lambda, n}^{I}$, where possibly $\min _{p \in P_{\lambda, n}^{I}} p \gg \lambda:=c(T-1)$, cf. (3.2), occurs. In order to allow for the use of smaller lattice sizes, we will weaken the requirements on the prime number sets $P^{I}$. To this end, we consider the set $I_{\bmod M}$, cf. (2.1), in more detail and define the sets

$$
\begin{aligned}
I_{\bmod M}^{\mathrm{u}}: & =\left\{\boldsymbol{h} \in I_{\bmod M}: \boldsymbol{h} \equiv \boldsymbol{k}_{1}(\bmod M), \boldsymbol{k}_{1} \in I, \text { and } \boldsymbol{h} \neq \boldsymbol{k}_{2}(\bmod M) \forall \boldsymbol{k}_{2} \in I \backslash\left\{\boldsymbol{k}_{1}\right\}\right\} \\
I_{\bmod M}^{\mathrm{c}}: & =\left\{\boldsymbol{h} \in I_{\bmod M}: \exists \boldsymbol{k}_{1}, \boldsymbol{k}_{2} \in I, \boldsymbol{k}_{1} \neq \boldsymbol{k}_{2}, \text { s.t. } \boldsymbol{h} \equiv \boldsymbol{k}_{1} \equiv \boldsymbol{k}_{2}(\bmod M)\right\} \\
& =I_{\bmod M \backslash I_{\bmod M}^{\mathrm{u}} .}
\end{aligned}
$$

The modulo $M$ operation on $I$ is a mapping from $I$ to $I_{\bmod M}$. Accordingly, the sets $I_{\bmod }^{\mathrm{u}} M$ consists of the images $\boldsymbol{h} \in I_{\bmod M}$ of all uniquely mapped vectors $\boldsymbol{k} \in I$ and the set $I_{\bmod }^{\mathrm{c}} M$ contains the vectors $\boldsymbol{h} \in I_{\bmod M}$ that are the images of at least two different $\boldsymbol{k}_{1}, \boldsymbol{k}_{2} \in I$, i.e., the images of vectors from $I$ that collides under the modulo operation. In addition, we define the sets $I_{M}^{\mathrm{u}}$ as the inverse image of $I_{\bmod M}^{\mathrm{u}}$.

Sampling a trigonometric polynomial $p \in \Pi_{I}$ at a rank-1 lattice of lattice size $M$ reads as

$$
p\left(\frac{j}{M} \boldsymbol{z}\right)=\sum_{\boldsymbol{k} \in I} \hat{p}_{\boldsymbol{k}} \mathrm{e}^{2 \pi \mathrm{i} \boldsymbol{k} \cdot \boldsymbol{z} \frac{j}{M}}=\sum_{\boldsymbol{k} \in I} \hat{p}_{\boldsymbol{k}} \mathrm{e}^{2 \pi \mathrm{i}(\boldsymbol{k} \bmod M) \cdot \boldsymbol{z} \frac{j}{M}}=\sum_{\boldsymbol{l} \in I \bmod M}\left(\sum_{\boldsymbol{l}=\boldsymbol{k} \in I} \hat{p}_{\boldsymbol{k}}\right) \mathrm{e}^{2 \pi \mathrm{i} \boldsymbol{l} \cdot \boldsymbol{z} \frac{j}{M}} .
$$


Accordingly, a reconstructing multiple rank-1 lattice for $I_{\bmod } M$ that consists of single rank-1 lattices of lattice sizes $M_{1}=\ldots=M_{s}=M$ allow for the unique reconstruction of all

$$
\hat{\tilde{p}}_{\boldsymbol{l}}:=\sum_{\substack{\boldsymbol{k} \in I \\ \boldsymbol{l}=\boldsymbol{k} \bmod M}} \hat{p}_{\boldsymbol{k}} .
$$

Obviously, if $\boldsymbol{k} \in I_{M}^{\mathrm{u}}$ holds, the sum at the right hand side of (4.5) contains exactly one summand and we achieve $\hat{p}_{\boldsymbol{k}}=\hat{\tilde{p}}_{\boldsymbol{k} \text { mod } M}$, i.e., we uniquely reconstruct all Fourier coefficients $\hat{p}_{\boldsymbol{k}}, \boldsymbol{k} \in I_{M}^{\mathrm{u}}$. If we observe $I_{\bmod M}^{\mathrm{c}} \neq \emptyset$, we cannot reconstruct all Fourier coefficients of $p \in \Pi_{I}$ in this way. However, a straightforward strategy similar to the approach in Section 4.1 can be applied. We consider the trigonometric polynomial

$$
p_{2}(\boldsymbol{x})=p(\boldsymbol{x})-\sum_{\boldsymbol{k} \in I_{M}^{\mathrm{u}}} \hat{p}_{\boldsymbol{k}} \mathrm{e}^{2 \pi \mathrm{i} \boldsymbol{k} \cdot \boldsymbol{x}}=\sum_{\boldsymbol{k} \in I \backslash I_{M}^{\mathrm{u}}} \hat{p}_{\boldsymbol{k}} \mathrm{e}^{2 \pi \mathrm{i} \boldsymbol{k} \cdot \boldsymbol{x}}
$$

in a next step.

At this point, we would like to mention that it is not necessary that the sets $I_{\bmod M_{j}}^{\mathrm{u}}$, $j=1, \ldots, \ell$ are non-empty in order to uniquely reconstruct all trigonometric polynomials with frequencies supported on $I$ using the multiple rank-1 lattice $\Lambda\left(z_{1}, M_{1}, \ldots, \boldsymbol{z}_{\ell}, M_{\ell}\right)$, which is illustrated in the following example.

Example 4.3. Let the frequency set

$$
I=\left\{\boldsymbol{k}_{1}, \ldots, \boldsymbol{k}_{6}\right\} \subset \mathbb{Z}^{d}
$$

with $k_{1, j}=\ldots=k_{6, j}, j=2, \ldots, d$, and $k_{j, 1}=(0,2,5,7,16,21)^{\top}$, i.e., the set $I$ is located on a line that is parallel to the first coordinate axis. We consider the multiple rank-1 lattice $\Lambda\left(\boldsymbol{z}_{1}, M_{1}, \boldsymbol{z}_{2}, M_{2}, \boldsymbol{z}_{3}, M_{3}\right)$ with $z_{j, 1}=1$ for $j=1,2,3$ and $M_{1}=2, M_{2}=3, M_{3}=5$, and we observe that $I_{\bmod M_{j}}^{\mathrm{u}}=\emptyset, j=1,2,3$, hold. Nevertheless, the Fourier matrix $\boldsymbol{A}\left(\Lambda\left(\boldsymbol{z}_{1}, M_{1}, \boldsymbol{z}_{2}, M_{2}, \boldsymbol{z}_{3}, M_{3}\right), I\right)$ has full column rank.

According to the last example, even the requirement $I_{\text {mod } M}^{\mathrm{u}} \neq \emptyset$ seems to restrict the considered rank-1 lattice sizes unnecessarily. However, the approaches of the last sections can easily deal with the sets $I_{\bmod M}$ and the requirement $I_{\bmod }^{\mathrm{u}} \neq \emptyset$ allows for a successive reduction of the frequency set $I$, cf. the context of (4.6).

\section{Bound the number of reconstructable Fourier coefficients from below}

One way to guarantee the success in using the aforementioned strategy is to ensure that $I_{\bmod M}^{\mathrm{u}}$ contains at least a significant number of frequencies, which only depends on $M$ for a given frequency set $I$. We search for the smallest prime number $M$ such that the number of frequencies within $I_{\bmod M}^{\mathrm{u}}$ fulfills $\left|I_{\bmod M}^{\mathrm{u}}\right| \geq \frac{|I|}{C}, C>1$, and $M>c\left(\left|I_{\bmod M}\right|-1\right), c>1$, i.e., we define

$$
M_{c}^{I, C}:=\min \left\{M \in \mathbb{N}: M \text { prime with }\left|I_{\bmod M}^{\mathrm{u}}\right| \geq \frac{|I|}{C} \text { and } M>c\left(\left|I_{\bmod M}\right|-1\right)\right\} .
$$

The application of one of the Algorithms 1 or 3 with fixed rank-1 lattice sizes $M$ allows for the direct reconstruction of all Fourier coefficients $\hat{p}_{\boldsymbol{k}}, \boldsymbol{k} \in I_{M}^{\mathrm{u}}$. Taking the last considerations as well as the iterative approach described in the context of (4.6) into account, we achieve 


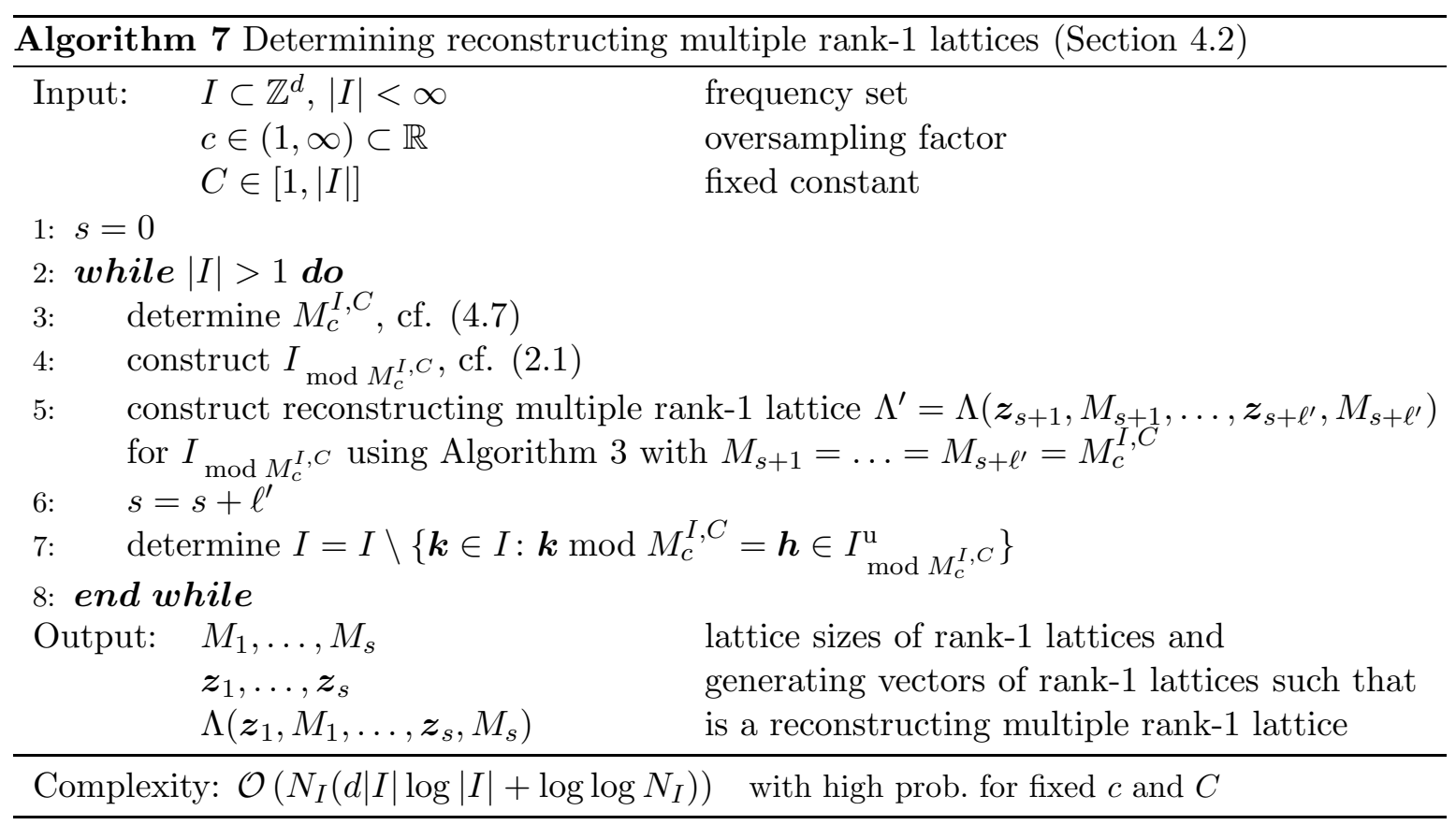

the search strategy for reconstructing multiple rank-1 lattices that is outlined in Algorithm 7. Similar to the considerations in Theorem 4.1, one realizes that the loop in Algorithm 7 is passed at most $\min \{C \log T, T\}$ times. Assuming that $M_{c}^{I, C}$ is in the magnitude of $T=|I|$, with a probability of at least $1-\delta$ the number of sampling nodes in the output of Algorithm 7 can simply be estimated by $T$ times a polylogarithmic term in $T$.

\subsection{Combining both strategies}

We can even combine the strategies described in Sections 4.1 and 4.2. In each step of the construction in Algorithm 7, we can choose the best of the determined $\ell^{\prime}$ rank-1 lattices that is used for a reconstruction. With high probability one of the rank-1 lattices allows for the unique reconstruction of $\frac{|I|}{C s}$ frequencies of $I$. Consequently, we can go straightforward in determining a reconstructing rank-1 lattice for a smaller frequency set of cardinality of at most $\left(1-\frac{1}{C s}\right)|I|$ frequencies, where $s$ depends on $c, \delta,|I|$, and $\left|I_{\bmod M}\right|$. Restricting the used prime lattice sizes $M$ being distinct from those that are already used in each step, we can also determine reconstructing multiple rank-1 lattices that are subsampling schemes of a huge single rank-1 lattice, cf. [10, Cor. 2.2].

\section{Numerical tests}

Since the complexity of the fast Fourier transform related to the reconstruction of the trigonometric polynomial mainly depends on the number of used sampling nodes, we focus on the oversampling factor $M /|I|$, i.e., the ratio of the number of used sampling nodes $M$ compared to the number $|I|$ of Fourier coefficients that will be reconstructed. In addition, the aforementioned complexity depends on the number $s$ of used single rank-1 lattices that are combined to a multiple rank-1 lattice. Consequently, we also present these values. Furthermore, we will compute some of the condition numbers of the Fourier matrices $\boldsymbol{A}\left(\Lambda\left(\boldsymbol{z}_{1}, M_{1}, \ldots, \boldsymbol{z}_{s}, M_{s}\right), I\right)$, 
cf. (1.3), that realizes the evaluation of the considered trigonometric polynomials, even though we do not have any theoretical statements on them. Due to the computational costs, we computed the condition numbers only for frequency sets of low cardinality, i.e., $|I| \leq 20000$.

A first numerical test on a very specific frequency set $I$ shows the advantages of Algorithm 7. Second, we consider random frequency sets and approve in some sense the universality of Algorithms 1 and 2. Last, we consider dyadic hyperbolic crosses as frequency sets $I$ and compare Algorithms 1 to 6. We leave out Algorithm 7 here, since we do not expect significant advantages due to the structure of the frequency sets.

\subsection{Specific frequency set}

We fix the parameters $\delta=0.5$ and $c=1.1$ and we consider the $d$-dimensional frequency set

$$
I:=\left\{\boldsymbol{k}_{1}, \ldots, \boldsymbol{k}_{5}\right\}=\left\{\left(\begin{array}{c}
0 \\
\boldsymbol{h}
\end{array}\right),\left(\begin{array}{c}
6251 \\
\boldsymbol{h}
\end{array}\right)\left(\begin{array}{c}
10879 \\
\boldsymbol{h}
\end{array}\right)\left(\begin{array}{c}
15457 \\
\boldsymbol{h}
\end{array}\right)\left(\begin{array}{c}
19499 \\
\boldsymbol{h}
\end{array}\right)\right\},
$$

where $\boldsymbol{h} \in \mathbb{Z}^{d-1}$ is fixed. Algorithms 1 and 2 will determine a multiple rank-1 lattice that contains at least one rank- 1 lattice of size $M_{1}=19501$ since this is the smallest prime number larger than $N_{I}=19499$. The smallest prime number within $P^{I}$ is 53. Accordingly, the outputs of Algorithms 3 to 6 will contain at least one rank-1 lattice of size $M_{1}=53$. Applying Algorithm 7 with $C=2$ determined $M_{1}=5$ and $M_{2}=3$ in each of our 10000 numerical tests, i.e., the determined reconstructing multiple rank- 1 lattices consist of only $M=1-2+5+3=7$ sampling nodes. The observed condition numbers $\operatorname{cond}(\boldsymbol{A})=\sqrt{\frac{19+\sqrt{21}}{11-\sqrt{61}}} \approx 2.7191$ of the Fourier matrices $\boldsymbol{A}$ were fixed.

\subsection{Random frequency sets}

Since Algorithms 1 and 2 only depend on the cardinality $T$ and the expansion $N_{I}$ of the considered frequency set $I$, we performed the following numerical test. We fix $c=2$ and $s=10,15,20,25,30$, and we compute $T=\left\lfloor\mathrm{e}^{\frac{s}{2}}\right\rfloor$, i.e., depending on $c=2$ and $s$ we choose $T$ as large as possible such that the estimate $1-\delta$ of the success probability in Theorem 3.4 is greater than zero. Accordingly, the statement of Theorem 3.4 ensures the construction of reconstructing multiple rank-1 lattices only with a low probability.

For each $s \in\{10,15,20,25,30\}$ we constructed exactly one reconstructing multiple rank-1 lattice for a frequency set $I$ with $N_{I}=299$ and $T_{s}=\left\lfloor\mathrm{e}^{\frac{s}{2}}\right\rfloor$. Subsequently, we determined 10000 randomly chosen frequency sets $I \subset[1,300]^{3}$ with $|I|=T_{s}$, where each frequency $\boldsymbol{k} \in I$ was chosen uniformly at random from $[1,300]^{3}$. For each of the frequency sets $I$ we checked the equality in (3.8), which guarantees a full column rank Fourier matrix and, thus, a unique reconstruction of all trigonometric polynomials in $\Pi_{I}$ by the means of the sampling values at the multiple rank-1 lattice. In Table 5.1, we collect the results and present the number of frequency sets $I$ that fulfilled the equality in (3.8). Due to the fact that we do not check the column rank of the Fourier matrices, the numbers in the column \# reco in Table 5.1 are only lower bounds on the number of frequency sets $I$, where the Fourier matrix $\boldsymbol{A}\left(\Lambda\left(\boldsymbol{z}_{1}, M_{1}, \ldots, \boldsymbol{z}_{s}, M_{s}\right), I\right)$ has full column rank.

We observe that the determined multiple rank-1 lattices are reconstructing multiple rank-1 lattices for at least $99 \%$ of the tested frequency sets $I$ in our numerical tests. We interpret this observation as a consequence of the rough estimates in our proofs. In detail, the multiple 


\begin{tabular}{|c|c|c|c|c|c|c|}
\hline & $T$ & $s$ & $1-\delta$ & $M$ & $M / T$ & \# reco \\
\hline \multirow{5}{*}{ 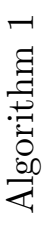 } & 148 & 10 & $2.784 \mathrm{e}-03$ & 3061 & $2.07 \mathrm{e}+01$ & 9904 \\
\hline & 1808 & 15 & $2.346 \mathrm{e}-05$ & 54241 & $3.00 \mathrm{e}+01$ & 9986 \\
\hline & 22026 & 20 & $2.115 \mathrm{e}-05$ & 881041 & $4.00 \mathrm{e}+01$ & 9999 \\
\hline & 268337 & 25 & $1.068 \mathrm{e}-06$ & 13416901 & $5.00 \mathrm{e}+01$ & 10000 \\
\hline & 3269017 & 30 & $1.139 \mathrm{e}-07$ & 196141261 & $6.00 \mathrm{e}+01$ & 10000 \\
\hline \multirow{5}{*}{ 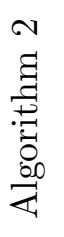 } & 148 & 10 & $2.784 \mathrm{e}-03$ & 3315 & $2.24 \mathrm{e}+01$ & 9951 \\
\hline & 1808 & 15 & $2.346 \mathrm{e}-05$ & 55061 & $3.05 \mathrm{e}+01$ & 9988 \\
\hline & 22026 & 20 & $2.115 \mathrm{e}-05$ & 882833 & $4.01 \mathrm{e}+01$ & 9999 \\
\hline & 268337 & 25 & $1.068 \mathrm{e}-06$ & 13420041 & $5.00 \mathrm{e}+01$ & 10000 \\
\hline & 3269017 & 30 & $1.139 \mathrm{e}-07$ & 196148197 & $6.00 \mathrm{e}+01$ & 10000 \\
\hline
\end{tabular}

Table 5.1: Applying Algorithms 1 and 2 for fixed $T, s$, and $c=2$, yielded $M=1-s+$ $\sum_{\ell=1}^{s} M_{\ell}$ and $M / T$. The resulting multiple rank-1 lattices have been tested for their reconstruction property against 10000 randomly chosen frequency sets $I \subset$ $[1,300]^{3}$ of cardinality $T$, where each $\boldsymbol{k} \in I$ was randomly and uniformly chosen in $[1,300]^{3} \cap \mathbb{Z}^{3}$. Column \#reco shows (a lower bound on) the number of frequency sets $I$, for which each $p \in \Pi_{I}$ was successfully reconstructable using the sampling values from the determined multiple rank-1 lattice.

application of union bounds affects strongly the estimate on the failure probability $\delta$, thus we expect that the considered approaches behave much better in practice.

\subsection{Dyadic hyperbolic crosses}

We consider dyadic hyperbolic crosses

$$
I=H_{n}^{d}:=\bigcup_{\|\boldsymbol{j}\|_{1}=n} G_{j_{1}} \times \ldots \times G_{j_{d}}, \quad G_{j}=\left(2^{j-1}, 2^{j-1}\right] \cap \mathbb{Z},
$$

as frequency sets $I$ and construct reconstructing multiple rank-1 lattices using Algorithms 1 to 6 with fixed parameters $c=2, \delta=0.5$. The parameters $T, d$, and $N$ in Algorithms 1 and 2 are determined by the frequency sets $H_{n}^{d}$. Similar to the considerations in [10], we focus on two different settings.

First we fix the dimension $d=6$ and increase the refinement $n$ up to twelve. The results are collected in the first rows of Table 5.3. In addition, Figure 5.1 shows the oversampling factors of the constructed multiple rank-1 lattices and some condition numbers. The constant slopes in the plots of the oversampling factors are caused by logarithmic dependencies on the cardinality of the frequency set $I$. Similar to the observations in [10] and even better than our theoretical results, the oversampling factors of the multiple rank-1 lattices that are constructed using Algorithms 5 and 6 seem to stagnate.

Second, we fix the refinements $n=2,3,4,5$ and consider growing dimension $d$. The lower rows of Table 5.3 presents some results of this numerical experiment. Figure 5.2 depicts the numerical tests in more detail.

In accordance to our theoretical results, we observe at most linearity in the oversampling factor $M / T$ with respect to $\log T$. Another interesting observation is that Algorithms 3 and 
Oversampling factors

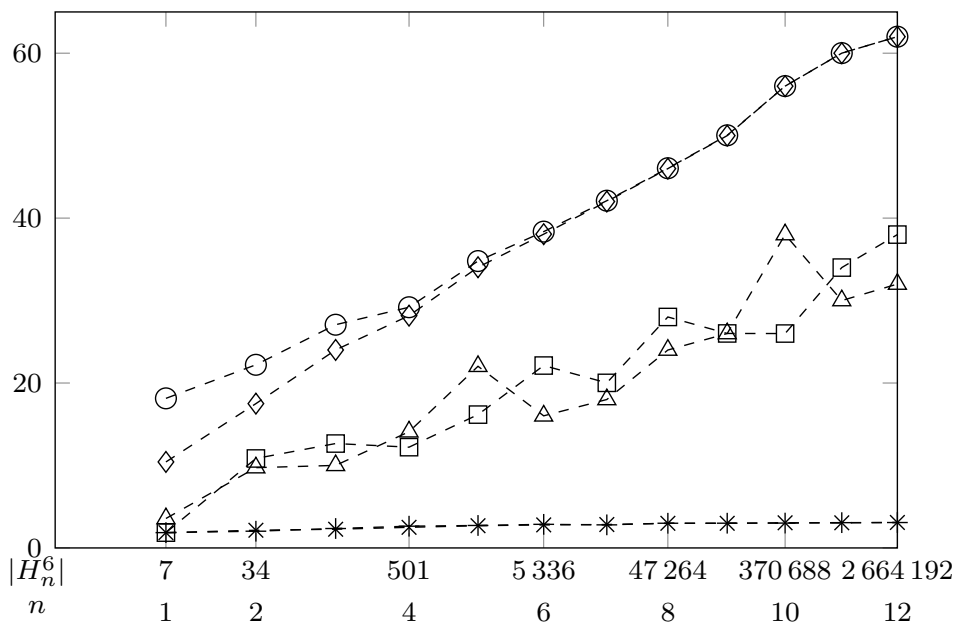

Condition numbers

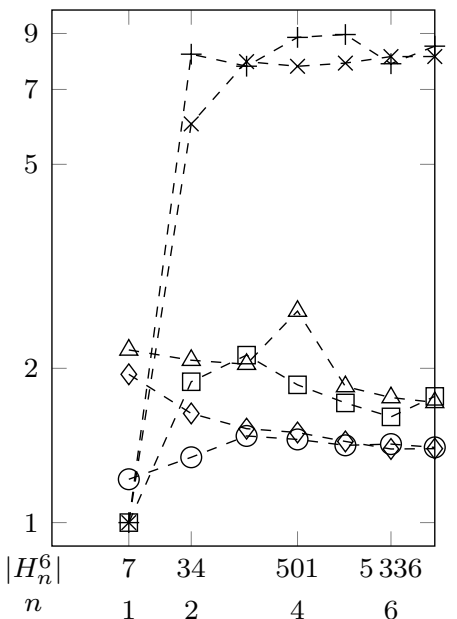

$-\diamond$ - Algorithm $1 \quad-\triangle$ - Algorithm $3 \quad-\rtimes$ - Algorithm 5

- - - Algorithm 2 - - Algorithm 4 - +- Algorithm 6

Figure 5.1: Oversampling factors of reconstructing multiple rank-1 lattices and the condition numbers of corresponding Fourier matrices for hyperbolic cross frequency sets $H_{n}^{6}$.

\begin{tabular}{l|ccccc}
$d$ & 6 & 50 & 42 & 18 & 10 \\
$n$ & 7 & 2 & 3 & 4 & 5 \\
$|I|$ & 16172 & 1376 & 16080 & 11644 & 8378 \\
$\operatorname{cond}(\boldsymbol{A})$ & $1.8 \mathrm{e} 4$ & $3.5 \mathrm{e} 5$ & $1.6 \mathrm{e} 7$ & $1.3 \mathrm{e} 6$ & $1.1 \mathrm{e} 5$
\end{tabular}

Table 5.2: Condition numbers of Fourier matrices $\boldsymbol{A}\left(\mathcal{X}, H_{n}^{d}\right), \mathcal{X}$ are the related sparse grids, for selected parameters $d$ and $n$.

4 constructs multiple rank-1 lattices of lower cardinality than Algorithm 1 and 2, which coincides with the considerations that led to Algorithms 3 and 4, cf. the context of (3.9). We observe that the application of Algorithms 5 and 6 result in the smallest oversampling factors in practice. The oversampling factors are quite small (less than 3.3) and seem to stagnate, which means that the oversampling factors behave notably better than the theoretical results. A similar behavior of the oversampling factors was already observed in [10].

As mentioned above, we additionally computed the condition numbers of the Fourier matrices $\boldsymbol{A}\left(\Lambda\left(\boldsymbol{z}_{1}, M_{1}, \ldots, \boldsymbol{z}_{s}, M_{s}\right), I\right)$, where the frequency sets $I$ are of cardinality up to 20000 , cf. Figures 5.1 and 5.3. We observed condition numbers that were bounded by two, three, and twelve using the multiple rank-1 lattices $\Lambda\left(\boldsymbol{z}_{1}, M_{1}, \ldots, \boldsymbol{z}_{s}, M_{s}\right)$ that are determined by Algorithms 1 and 2, Algorithms 3 and 4, and Algorithms 5 and 6, respectively. In general, the tests does not allow for an reliable interpretation. Nevertheless, we suppose that the condition numbers do not substantially increase for growing cardinality of the frequency set $I$.

At this point, we would like to compare the obtained numerical results to those for dyadic sparse grids, since these are the natural spatial discretizations for the considered dyadic hyperbolic crosses in frequency domain, cf. [6]. Due to the correlated construction of dyadic sparse grids and dyadic hyperbolic crosses, the oversampling factor is exactly one and we 

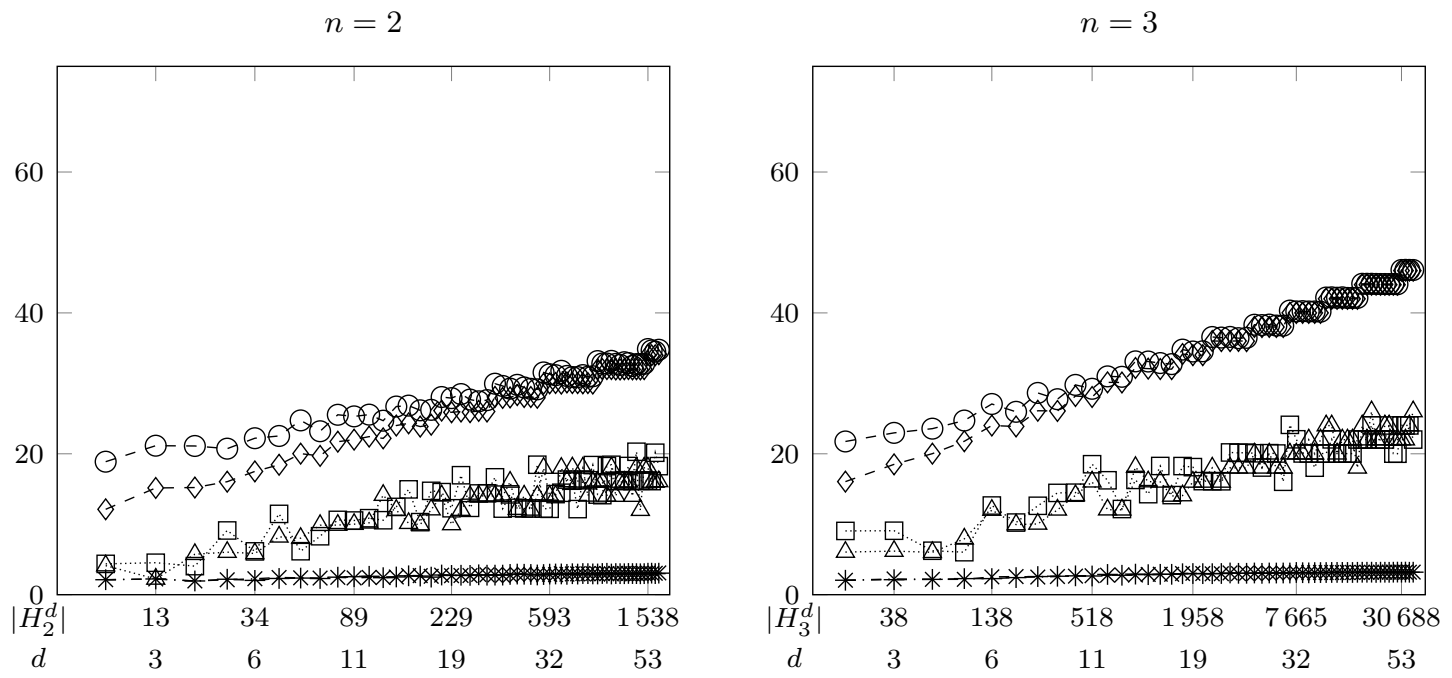

$$
n=4
$$
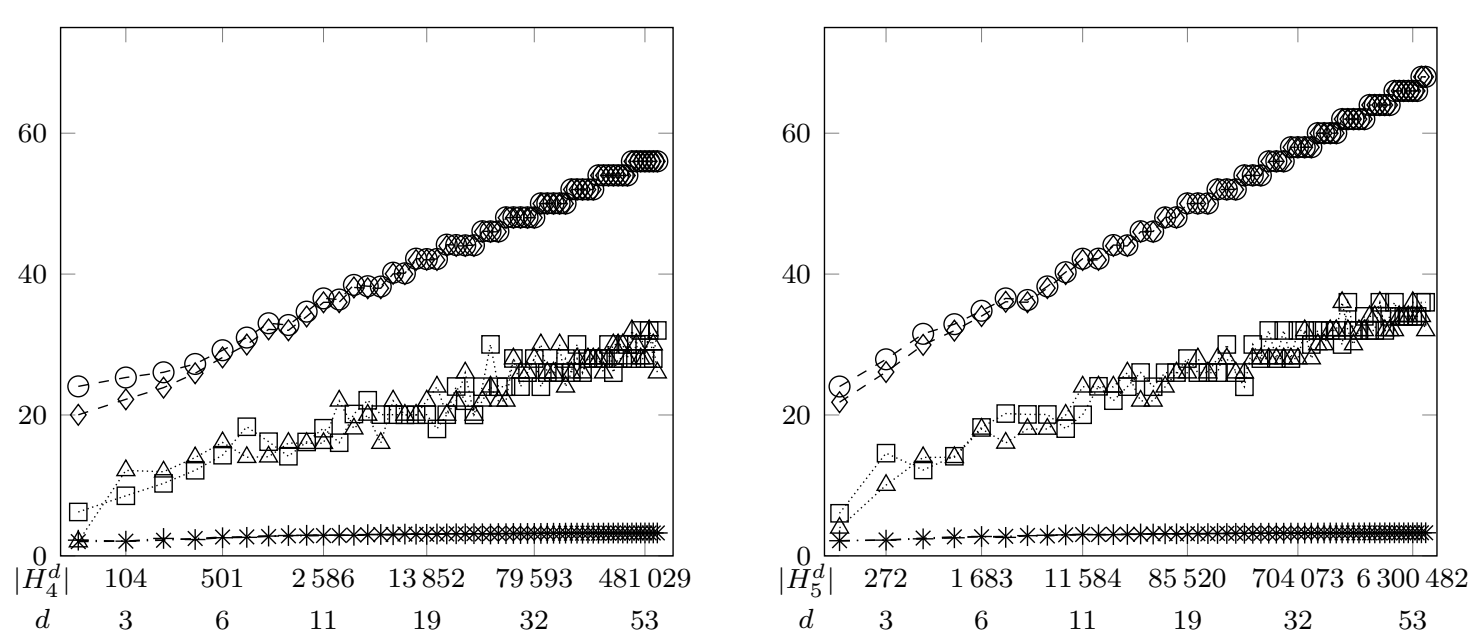

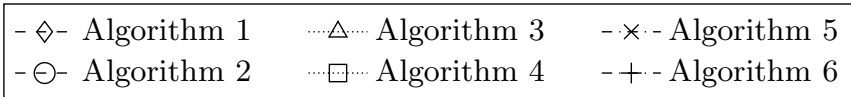

Figure 5.2: Oversampling factors of reconstructing multiple rank-1 lattices for hyperbolic cross frequency sets $H_{n}^{d}, n=2,3,4,5$ fixed.

focus on the condition number, which was investigated in [11]. Therein, the authors showed lower bounds on the condition numbers of the Fourier matrices $\boldsymbol{A}\left(\mathcal{X}, H_{n}^{d}\right)$, cf. (1.1), where the spatial discretizations $\mathcal{X}$ are the related sparse grids. These bounds increase for growing matrix dimensions, i.e., for growing refinement $n$ and growing spatial dimension $d$, cf. [11] for details. Besides the theoretical considerations, numerically determined condition numbers were presented. Some of them are stated in Table 5.2. Even for matrices of less than 20000 columns, one computes condition numbers up to several millions. The numerically determined, outstanding condition numbers for multiple rank-1 lattice spatial discretizations are substantially lower, cf. rows $9,21,27,31,37$ of Table 5.3, and consequently, preferable. 


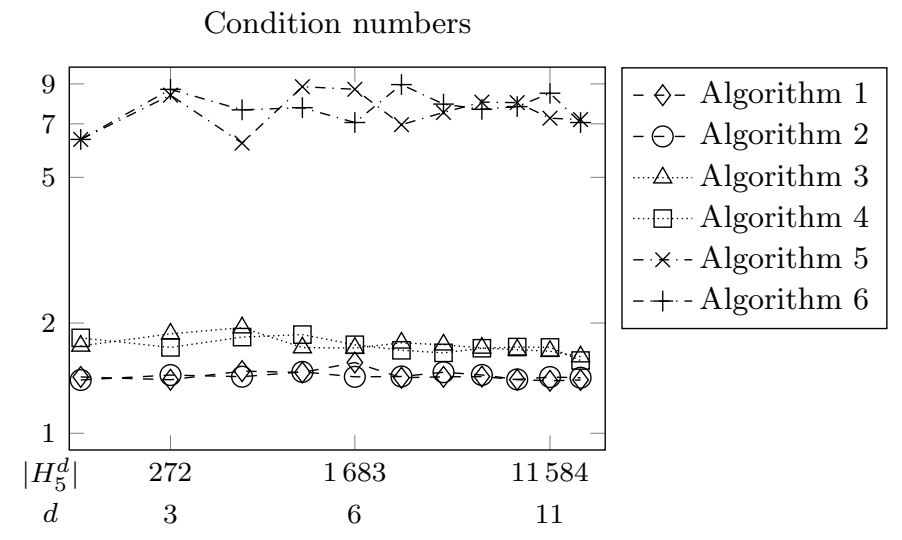

Figure 5.3: Condition numbers of Fourier matrices $\boldsymbol{A}\left(\Lambda\left(\boldsymbol{z}_{1}, M_{1}, \ldots, \boldsymbol{z}_{s}, M_{s}\right), H_{5}^{d}\right)$, $d=2, \ldots, 12$, for different reconstructing multiple rank-1 lattices $\Lambda\left(\boldsymbol{z}_{1}, M_{1}, \ldots, \boldsymbol{z}_{s}, M_{s}\right)$.

\section{Conclusion}

The concept of sampling schemes that consists of a set of rank-1 lattices allows for the reconstruction of multivariate trigonometric polynomials with frequencies supported on arbitrary frequency sets $I \subset \mathbb{Z}^{d}, N_{I} \lesssim|I|$. The oversampling factor, i.e., the ratio of the required number of used sampling values to the number of frequencies, is bounded by terms that are logarithmic in the number of frequencies with a very high probability.

Numerical tests, cf. also [10], indicate that even the condition numbers of the corresponding Fourier matrices are bounded, which is in fact the crucial remaining unproved characteristic. Reasonable bounds on these condition numbers that coincide roughly with the numerical observations might imply excellent approximation properties and even a highly improved Marcinkiewicz-type theorem for hyperbolic cross trigonometric polynomials - at least in $L_{2}$, cf. [2] and [21], respectively.

\section{Acknowledgments}

The author thanks the referees for the valuable comments and suggestions and gratefully acknowledges the funding by the Deutsche Forschungsgemeinschaft (DFG, German Research Foundation) - 380648269 .

\section{References}

[1] A. Arnold, M. Giesbrecht, and D. S. Roche. Faster sparse multivariate polynomial interpolation of straight-line programs. J. Symbolic Comput., 75:4-24, 2016. Special issue on the conference ISSAC 2014: Symbolic computation and computer algebra.

[2] G. Byrenheid, L. Kämmerer, T. Ullrich, and T. Volkmer. Tight error bounds for rank-1 lattice sampling in spaces of hybrid mixed smoothness. Numer. Math., 136:993-1034, 2017.

[3] R. Cools and D. Nuyens. An overview of fast component-by-component constructions of lattice rules and lattice sequences. PAMM, 7:1022609-1022610, 2007.

[4] D. Dũng, V. N. Temlyakov, and T. Ullrich. Hyperbolic cross approximation. ArXiv e-prints, Dec. 2016. arXiv:1601.03978 [math.NA]. 
[5] K. Gröchenig, B. M. Pötscher, and H. Rauhut. Learning trigonometric polynomials from random samples and exponential inequalities for eigenvalues of random matrices. arXiv:math/0701781v2, 2010.

[6] K. Hallatschek. Fouriertransformation auf dünnen Gittern mit hierarchischen Basen. Numer. Math., 63:83-97, 1992.

[7] W. Hoeffding. Probability inequalities for sums of bounded random variables. J. Amer. Statist. Assoc., 58:13 - 30, 1963.

[8] L. Kämmerer. Reconstructing hyperbolic cross trigonometric polynomials by sampling along rank-1 lattices. SIAM J. Numer. Anal., 51:2773-2796, 2013.

[9] L. Kämmerer. High Dimensional Fast Fourier Transform Based on Rank-1 Lattice Sampling. Dissertation. Universitätsverlag Chemnitz, 2014.

[10] L. Kämmerer. Multiple rank-1 lattices as sampling schemes for multivariate trigonometric polynomials. J. Fourier Anal. Appl., 2016.

[11] L. Kämmerer and S. Kunis. On the stability of the hyperbolic cross discrete Fourier transform. Numer. Math., 117:581-600, 2011 .

[12] L. Kämmerer, D. Potts, and T. Volkmer. Approximation of multivariate periodic functions by trigonometric polynomials based on rank-1 lattice sampling. J. Complexity, 31:543-576, 2015.

[13] L. Kämmerer, D. Potts, and T. Volkmer. Approximation of multivariate periodic functions by trigonometric polynomials based on sampling along rank-1 lattice with generating vector of Korobov form. J. Complexity, $31: 424-456,2015$

[14] N. M. Korobov. On the approximate computation of multiple integrals. Dokl. Akad. Nauk, 124:1207-1210, 1959. In Russian.

[15] N. M. Korobov. Number-Theoretic Methods in Approximate Analysis. Fizmatgiz, Moscow, 1963. In Russian.

[16] F. Y. Kuo, I. H. Sloan, and H. Woźniakowski. Lattice rules for multivariate approximation in the worst case setting. In H. Niederreiter and D. Talay, editors, Monte Carlo and Quasi-Monte Carlo Methods 2004, pages 289-330. Springer Berlin Heidelberg, Berlin, 2006.

[17] H. Niederreiter. Quasi-Monte Carlo methods and pseudo-random numbers. B. Am. Math. Soc., 84:957-1041, 1978.

[18] J. B. Rosser and L. Schoenfeld. Approximate formulas for some functions of prime numbers. Illinois J. Math., 6:64 - 94, 031962.

[19] I. H. Sloan and A. V. Reztsov. Component-by-component construction of good lattice rules. Math. Comp., 71:263$273,2002$.

[20] V. N. Temlyakov. Reconstruction of periodic functions of several variables from the values at the nodes of numbertheoretic nets. Anal. Math., 12:287-305, 1986. In Russian.

[21] V. N. Temlyakov. The Marcinkiewicz-type discretization theorems for the hyperbolic cross polynomials. ArXiv e-prints, 2017. arXiv:1702.01617 [math.NA].

[22] C. F. Van Loan. Computational Frameworks for the Fast Fourier Transform. SIAM, Philadelphia, PA, USA, 1992. 


\begin{tabular}{|c|c|c|c|c|c|c|c|c|c|c|c|c|c|c|c|c|c|c|c|c|c|}
\hline & \multirow[b]{2}{*}{$d$} & \multirow[b]{2}{*}{$n$} & \multirow[b]{2}{*}{$|I|$} & \multicolumn{3}{|c|}{ Algorithm 1} & \multicolumn{3}{|c|}{ Algorithm 2} & \multicolumn{3}{|c|}{ Algorithm 3} & \multicolumn{3}{|c|}{ Algorithm 4} & \multicolumn{3}{|c|}{ Algorithm 5} & \multicolumn{3}{|c|}{ Algorithm 6} \\
\hline & & & & $s$ & $M /|I|$ & $\operatorname{cond}(\boldsymbol{A})$ & $s$ & $M /|I|$ & $\operatorname{cond}(\boldsymbol{A})$ & $\tilde{s}$ & $M /|I|$ & $\operatorname{cond}(\boldsymbol{A})$ & $\tilde{s}$ & $M /|I|$ & $\operatorname{cond}(\boldsymbol{A})$ & $\ell$ & $M /|I|$ & $\operatorname{cond}(\boldsymbol{A})$ & $\ell$ & $M /|I|$ & $\operatorname{cond}(\boldsymbol{A})$ \\
\hline \multirow{12}{*}{ 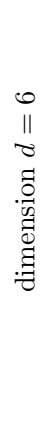 } & 6 & 1 & 7 & 6 & 10.4 & 1.9 & 6 & 18.1 & 1.2 & 2 & 3.6 & 2.2 & 1 & 1.9 & 1.0 & 1 & 1.9 & 1.0 & 1 & 1.9 & 1.0 \\
\hline & 6 & 2 & 34 & 9 & 17.5 & 1.6 & 9 & 22.2 & 1.3 & 5 & 9.7 & 2.1 & 5 & 10.9 & 1.9 & 2 & 2.1 & 6.0 & 2 & 2.0 & 8.2 \\
\hline & 6 & 3 & 138 & 12 & 24.0 & 1.5 & 12 & 27.1 & 1.5 & 5 & 10.0 & 2.0 & 6 & 12.7 & 2.1 & 3 & 2.3 & 7.9 & 3 & 2.4 & 7.8 \\
\hline & 6 & 4 & 501 & 14 & 28.2 & 1.5 & 14 & 29.2 & 1.5 & 7 & 14.1 & 2.6 & 6 & 12.2 & 1.9 & 4 & 2.5 & 7.8 & 4 & 2.6 & 8.8 \\
\hline & 6 & 5 & 1683 & 17 & 34.0 & 1.4 & 17 & 34.8 & 1.4 & 11 & 22.0 & 1.8 & 8 & 16.2 & 1.7 & 5 & 2.7 & 7.9 & 5 & 2.7 & 9.0 \\
\hline & 6 & 6 & 5336 & 19 & 38.1 & 1.4 & 19 & 38.4 & 1.4 & 8 & 16.0 & 1.8 & 11 & 22.1 & 1.6 & 6 & 2.8 & 8.1 & 6 & 2.9 & 7.9 \\
\hline & 6 & 7 & 16172 & 21 & 42.0 & 1.4 & 21 & 42.1 & 1.4 & 9 & 18.0 & 1.7 & 10 & 20.0 & 1.8 & 7 & 2.8 & 8.1 & 7 & 2.8 & 8.5 \\
\hline & 6 & 8 & 47264 & 23 & 46.0 & - & 23 & 46.1 & - & 12 & 24.0 & - & 14 & 28.0 & - & 9 & 3.0 & - & 8 & 3.0 & - \\
\hline & 6 & 9 & 134048 & 25 & 50.0 & - & 25 & 50.0 & - & 13 & 26.0 & - & 13 & 26.0 & - & 10 & 3.0 & - & 10 & 3.0 & - \\
\hline & 6 & 10 & 370688 & 28 & 56.0 & - & 28 & 56.0 & - & 19 & 38.0 & - & 13 & 26.0 & - & 11 & 3.0 & - & 11 & 3.0 & - \\
\hline & 6 & 11 & 1003136 & 30 & 60.0 & - & 30 & 60.0 & - & 15 & 30.0 & - & 17 & 34.0 & - & 12 & 3.0 & - & 12 & 3.1 & - \\
\hline & 6 & 12 & 2664192 & 31 & 62.0 & - & 31 & 62.0 & - & 16 & 32.0 & - & 19 & 38.0 & - & 13 & 3.1 & - & 13 & 3.1 & - \\
\hline \multirow{7}{*}{ 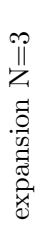 } & 2 & 2 & 8 & 6 & 12.1 & 1.5 & 6 & 18.9 & 1.2 & 2 & 4.1 & 1.7 & 2 & 4.4 & 1.7 & 1 & 2.1 & 1.0 & 1 & 2.1 & 1.0 \\
\hline & 10 & 2 & 76 & 11 & 21.7 & 1.7 & 11 & 25.5 & 1.7 & 5 & 9.9 & 2.1 & 5 & 10.6 & 1.9 & 2 & 2.5 & 4.6 & 3 & 2.5 & 6.8 \\
\hline & 18 & 2 & 208 & 13 & 26.1 & 1.5 & 13 & 28.1 & 1.6 & 7 & 14.1 & 1.9 & 7 & 14.6 & 1.8 & 4 & 2.8 & 7.3 & 3 & 2.8 & 6.2 \\
\hline & 26 & 2 & 404 & 14 & 28.0 & 1.5 & 14 & 29.3 & 1.5 & 8 & 16.0 & 1.8 & 7 & 14.2 & 1.9 & 4 & 2.8 & 6.7 & 4 & 2.9 & 7.9 \\
\hline & 34 & 2 & 664 & 15 & 30.0 & 1.5 & 15 & 31.8 & 1.5 & 9 & 18.0 & 1.7 & 7 & 14.5 & 1.8 & 5 & 3.0 & 6.7 & 5 & 2.9 & 6.9 \\
\hline & 42 & 2 & 988 & 16 & 32.0 & 1.4 & 16 & 32.8 & 1.5 & 8 & 16.0 & 1.8 & 7 & 14.1 & 1.9 & 5 & 3.0 & 6.9 & 5 & 3.0 & 7.2 \\
\hline & 50 & 2 & 1376 & 16 & 32.0 & 1.5 & 16 & 32.7 & 1.5 & 9 & 18.0 & 1.7 & 10 & 20.3 & 1.7 & 5 & 3.0 & 6.8 & 5 & 3.0 & 7.8 \\
\hline \multirow{7}{*}{ 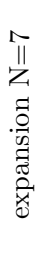 } & 2 & 3 & 20 & 8 & 16.1 & 1.4 & 8 & 21.8 & 1.4 & 3 & 6.0 & 1.7 & 4 & 9.1 & 1.7 & 1 & 2.0 & 1.0 & 1 & 2.0 & 1.0 \\
\hline & 10 & 3 & 416 & 14 & 28.2 & 1.4 & 14 & 29.8 & 1.6 & 7 & 14.1 & 1.9 & 7 & 14.5 & 1.7 & 4 & 2.6 & 7.5 & 4 & 2.7 & 9.5 \\
\hline & 18 & 3 & 1708 & 17 & 34.2 & 1.4 & 17 & 34.8 & 1.5 & 7 & 14.1 & 1.9 & 9 & 18.3 & 1.7 & 6 & 3.0 & 7.1 & 6 & 3.0 & 7.7 \\
\hline & 26 & 3 & 4408 & 19 & 38.0 & 1.4 & 19 & 38.3 & 1.4 & 10 & 20.0 & 1.7 & 10 & 20.1 & 1.7 & 6 & 3.0 & 7.5 & 6 & 3.1 & 7.0 \\
\hline & 34 & 3 & 9028 & 20 & 40.0 & 1.4 & 20 & 40.2 & 1.4 & 11 & 22.0 & 1.6 & 10 & 20.0 & 1.7 & 7 & 3.1 & 7.2 & 7 & 3.1 & 6.8 \\
\hline & 42 & 3 & 16080 & 21 & 42.0 & 1.4 & 21 & 42.1 & 1.4 & 11 & 22.0 & 1.7 & 10 & 20.0 & 1.7 & 8 & 3.2 & 7.4 & 8 & 3.2 & 7.2 \\
\hline & 50 & 3 & 26076 & 22 & 44.0 & - & 22 & 44.1 & - & 12 & 24.0 & - & 12 & 24.0 & - & 9 & 3.2 & - & 8 & 3.2 & - \\
\hline \multirow{7}{*}{ 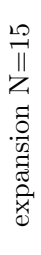 } & 2 & 4 & 48 & 10 & 20.0 & 1.4 & 10 & 24.1 & 1.4 & 1 & 2.0 & 1.0 & 3 & 6.2 & 1.9 & 1 & 2.0 & 1.0 & 2 & 2.2 & 5.4 \\
\hline & 10 & 4 & 1966 & 17 & 34.0 & 1.5 & 17 & 34.7 & 1.4 & 8 & 16.0 & 1.9 & 8 & 16.2 & 1.9 & 6 & 2.8 & 7.4 & 5 & 2.9 & 6.3 \\
\hline & 18 & 4 & 11644 & 21 & 42.0 & 1.4 & 21 & 42.2 & 1.4 & 10 & 20.0 & 1.7 & 10 & 20.0 & 1.8 & 7 & 3.1 & 7.0 & 7 & 3.1 & 7.5 \\
\hline & 26 & 4 & 39066 & 23 & 46.0 & - & 23 & 46.1 & - & 12 & 24.0 & - & 15 & 30.0 & - & 9 & 3.2 & - & 9 & 3.1 & - \\
\hline & 34 & 4 & 98312 & 25 & 50.0 & - & 25 & 50.0 & - & 13 & 26.0 & - & 13 & 26.0 & - & 10 & 3.2 & - & 10 & 3.2 & - \\
\hline & 42 & 4 & 207558 & 26 & 52.0 & - & 26 & 52.0 & - & 14 & 28.0 & - & 14 & 28.0 & - & 10 & 3.2 & - & 10 & 3.2 & - \\
\hline & 50 & 4 & 389076 & 28 & 56.0 & - & 28 & 56.0 & - & 16 & 32.0 & - & 14 & 28.0 & - & 11 & 3.2 & - & 11 & 3.2 & - \\
\hline \multirow{7}{*}{ 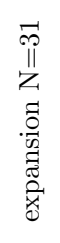 } & 2 & 5 & 112 & 11 & 21.8 & 1.4 & 11 & 24.0 & 1.4 & 2 & 4.0 & 1.7 & 3 & 6.0 & 1.8 & 2 & 2.2 & 6.3 & 2 & 2.2 & 6.3 \\
\hline & 10 & 5 & 8378 & 20 & 40.0 & 1.4 & 20 & 40.3 & 1.4 & 10 & 20.0 & 1.7 & 9 & 18.1 & 1.7 & 7 & 3.0 & 8.0 & 7 & 3.0 & 7.8 \\
\hline & 18 & 5 & 69460 & 24 & 48.0 & - & 24 & 48.1 & - & 13 & 26.0 & - & 13 & 26.0 & - & 9 & 3.1 & - & 9 & 3.1 & - \\
\hline & 26 & 5 & 297662 & 27 & 54.0 & - & 27 & 54.0 & - & 14 & 28.0 & - & 15 & 30.0 & - & 11 & 3.2 & - & 11 & 3.2 & - \\
\hline & 34 & 5 & 909688 & 29 & 58.0 & - & 29 & 58.0 & - & 14 & 28.0 & - & 15 & 30.0 & - & 12 & 3.3 & - & 12 & 3.2 & - \\
\hline & 42 & 5 & 2257410 & 31 & 62.0 & - & 31 & 62.0 & - & 16 & 32.0 & - & 16 & 32.0 & - & 13 & 3.3 & - & 13 & 3.3 & - \\
\hline & 50 & 5 & 4860636 & 33 & 66.0 & - & 33 & 66.0 & - & 17 & 34.0 & - & 17 & 34.0 & - & 14 & 3.3 & - & 14 & 3.3 & - \\
\hline
\end{tabular}

Table 5.3: Numbers $s, \tilde{s}, \ell$ of joined rank-1 lattices for the construction of reconstructing multiple rank-1 lattices for hyperbolic cross frequency sets of different dimensions $d$ and refinements $n$ using Algorithms 1 to 6 ; oversampling factors $M /|I|$ and condition numbers $\operatorname{cond}(\boldsymbol{A})$ of corresponding Fourier matrices, parameters $c=2$ and $\delta=0.5$ fixed. 